\title{
«De la part d'un ami sans visage». Agostino J. Sinadino (1876-1956), un poète sans profil: Correspondance inédite avec André Gide
}

\section{Paul-André Claudel}

\section{(2) OpenEdition Journals}

Édition électronique

URL : http://journals.openedition.org/studifrancesi/34322

DOI : 10.4000/studifrancesi.34322

ISSN : 2421-5856

Éditeur

Rosenberg \& Sellier

\section{Édition imprimée}

Date de publication : 1 décembre 2005

Pagination : $565-598$

ISSN : 0039-2944

\section{Référence électronique}

Paul-André Claudel, « «De la part d'un ami sans visage». Agostino J. Sinadino (1876-1956), un poète sans profil: Correspondance inédite avec André Gide », Studi Francesi [En ligne], 147 (XLX | III) | 2005, mis en ligne le 30 novembre 2015, consulté le 19 avril 2021. URL : http://journals.openedition.org/ studifrancesi/34322 ; DOI : https://doi.org/10.4000/studifrancesi.34322

\section{(c)}

Studi Francesi è distribuita con Licenza Creative Commons Attribuzione - Non commerciale - Non opere derivate 4.0 Internazionale. 


\section{"De la part d'un ami sans visage» Agostino J. Sinadino (1876-1956), un poète sans profil Correspondance inédite avec André Gide}

L'idéal de transparence documentaire se heurte, dans le cas du poète Agostino J. Sinadino, à une irréductible opacité. Depuis les quelques pages de présentation que Gian Pietro Lucini lui consacrait dans Il verso libero (1908), où Sinadino - décrit tour à tour comme un personnage de conte fantastique atteint d'une douce folie, un esthète menant une vie de bohème, un aventurier lancé sur les traces de Rimbaud, le résultat d'un croisement discutable entre Salomé et un prince des Mille et une nuits - finissait par se dissoudre totalement dans un imaginaire romanesque bien loin de la réalité ${ }^{1}$, jusqu'à la notice d'une grande sécheresse rédigée par Glauco Viazzi pour son anthologie des poèmes de Sinadino (1972) - où le critique reconnaissait ne savoir «pratiquement rien» de l'existence du poète ${ }^{2}-$, c'est le caractère fondamentalement impénétrable de Sinadino qui retient - et peut-être paralyse - l'attention critique.

L'effacement, la réduction des données touchent tous les types de documents qui concernent Sinadino, des fiches d'état civil qui enregistrent sa naissance jusqu'à la trace qui habituellement a la fonction sacrée de résumer la vie d'un individu: le nom du poète n'apparaît même pas sur la dalle qui couvre sa sépulture, dans la galerie du Cimitero Monumentale où repose son corps. L'absence de contours de cette figure énigmatique - qui rappelle la «réfringence» que Valéry avait observée chez l'insaisissable Mallarmé , ou le «vide intérieur» que Sartre croyait déceler dans la

(1) Voici comment Gian Pietro Lucini présente la figure de Sinadino, dans son essai de 1908: «Visse nel frastuono di una grande città e gli parve di vegetare tra le ombre frenetiche di un girone dantesco; poi, nel silenzio selvaggio di un castelletto smantellato sulle montagne sabine, con un cane, [una] bellissima moglie, un grande organo e molte fantasime. Oggi, mi dicono abbia passato il mare, commesso o segretario di qualche banca newyorkese, sempre colla medesima incoscienza, seminator di armonie sospese e diffuse, anima rapita nel turbine della vita moderna, catalessi in un sogno, in cui vede roteare il mondo in nebulosi, come le creature della sua poetica, forse felice; pargolo di mista razza, nato da una bella danzatrice milanese e da un ricco pascià levantino, greco d'Alessandria d'Egitto, prodotto incompleto, ma degno di studio e di qualche considerazione, nella categoria dei grotteschi geniali e delli innocenti e formidabili sgretolatori di retoriche e di leggi ch'io abbia mai conosciuto; prodigio e demenza» (G. P. Lucini, Ragion poetica e programma del verso libero. Grammatica, ricordi e confidenze per servire alla storia delle lettere contemporanee, Milano, Edizioni di Poesia, 1908, pp. 610-611).

(2) Dans la Giustificazione bibliografica qui apparaît en fin de volume, Glauco Viazzi reconnaît: «Di Agostino J. Sinadinò praticamente non si sa nulla, eccezion fatta per quanto ne scrisse Gian Pietro Lucini nel Verso libero [...]. Stampati in edizioni a tiratura limitatissima, quando non fuori commercio, e solitamente assenti nelle pubbliche biblioteche, i libri del Sinadinò sono difficilissimi da reperire» (A. J. Sinadinò, Poesie, a cura di Glauco Viazzi, Napoli, Guida, 1972, p. 161).

(3) «Ce qu’on nomme leur obscurité n'est, en vérité, 
personne de Baudelaire ${ }^{4}$ - produit un véritable brouillage de l'existence objective, et en multiplie les efflorescences imaginaires. «Tous les traits qui composent son image, il faudra donc les concevoir comme affectés d'un néant subtil et secret», nous avertit Sartre. Nous pourrions continuer avec lui, en reprenant sa méthodepour l'appliquer à Sinadino: «et tous les mots dont nous userons pour le peindre, il faudra éviter d'en être dupes car il évoquent et suggèrent beaucoup plus qu'il n'était; rappelons-nous, si nous voulons entrevoir les paysages lunaires de cette âme désolée, qu'un homme n'est jamais qu'une imposture»".

\section{Une biographie-labyrinthe}

La biographie de Sinadino, loin de se développer selon un parcours linéaire, se perd entre les pays et les langues, plus précisément entre les quatre déserts - réels ou métaphoriques - que sont l'Égypte, l'Italie, la France et les Etats-Unis. Né le 15 février 1876 au Caire, Sinadino est le fils d'un aventurier de la finance d'origine grecque accointé au milieu anglophone (d'où le «Sinadino» et le second prénom «John») et d'une musicienne milanaise de bonne famille (à qui il doit le très italien «Agostino»). Sa formation, son existence tout entière, gardent le signe indélébile de l'Egypte, et surtout d'Alexandrie, dont il intègre à la fois le cosmopolitisme aristocratique et le plurilinguisme $e^{6}$. Après la mort de son père (1890), les traces de Sinadino se perdent entre les deux rives de la Méditerranée: Milan et le Lac Majeur d'un côté, Alexandrie et Le Caire de l'autre. L'entrée de Sinadino dans le monde des lettres remonte en tous les cas à la fin du siècle: le poète publie entre Alexandrie, Milan et Lugano une série de quatre plaquettes $(1898-1901)^{7}$ qui constituent le cœur de sa première saison littéraire, et l'introduisent dans le cercle de Gian Pietro Lucini. Après un mariage célébré à Londres en 1900, Sinadino s'installe quelques années au sud de Rome, et participe à l'aventure de la Revue du Nord dirigée par son ami Giuseppe Vannicola. En 1906 il abandonne soudain l'Italie, sous la menace d'un créancier trop insistant, pour commencer une nouvelle vie à New York. Il semble avoir abandonné la poésie et s'être noyé dans l'univers capitaliste américain. Certains témoignages sur le séjour de Sinadino aux États-Unis évoquent la fondation d'une banque, en société avec d'autres Italiens, et sa faillite presque immédiate pour cause de gestion calamiteuse.

Quelques années plus tard, on retrouve la trace de Sinadino en Égypte (1910). Le poète, revenu à Alexandrie, a repris l'écriture et les publications.Dans la prestigieuse revue Grammata, son nom apparaît à quatre reprises, associé à une nouvelle manière, qui peut être considérée comme typique de sa deuxième saison littéraire ${ }^{8}$.

que leur réfringence», disait Paul Valéry des «systèmes cristallins» de Mallarmé (Cf. P. VALÉRY, Lettre sur Mallarmé, in Variété, II, in Euvres complètes, Paris, Gallimard, coll. «La Pléiade», 1957, vol. I, p. 639).

(4) «Il faut plutôt concevoir que les sentiments de Baudelaire ont une sorte de vide intérieur» (J.-P. SARTRE, Baudelaire, Paris, Gallimard, coll. «Folio Essais», 1994, p. 77).

(5) Ibid., p. 77.

(6) Sinadino a pu développer pendant son enfance une excellente connaissance de l'italien (sa langue maternelle), mais aussi du français (la langue des échanges culturels à Alexandrie) et de l'anglais (lié au milieu des grands investisseurs, fréquenté par son père); de nombreux documents témoignent de ce trilinguisme actif du poète. Par sa naissance dans la parikia, Sinadino maîtrise sans doute, de surcroît, quelques rudiments de grec, ce qui porte sa compétence à quatre langues. Sa nationalité "officielle" est d'ailleurs la nationalité grecque (Sinadino signe occasionnellement ses lettres «le byzantin», et l'accent qui vient parfois se poser sur la dernière syllabe de son nom de famille semble rappeler sa prononciation en grec).

(7) Respectivement: A. J. SinADINO, Le presenze invisibili, Alessandria d'Egitto, Cartoleria \& Tipografia Albert Zoller, 1898; A. G. SINADINÒ, La donna dagli specchi. Con un frontispizio di Carlo Agazzi, Milano, Stabilimento tipografico Bassi \& Protti, 1899; A. G. Sinadinò, Melodie, Lugano, Stamperia del Tessin-Touriste, 1900; A. G. SinADINÒ, Solennità: la festa, Lugano, Stamperia del Tessin-Touriste, 1901.

(8) À Alexandrie, Sinadino publie dès 1910 une 
Sinadino ne résiste pas, cependant, à la tentation européenne. Il réapparaît en Italie (1924), puis en France (1929), où il publie un élégant recueil grâce au soutien d'André Gide': troisième étape de l'écriture, sur le versant français cette fois. Mais l'année 1930, marquée par le double décès de la mère et du frère de Sinadino, met fin à l'époque des grands voyages et de la prodigalité. Sans l'appui charitable de la famille proche, habituée à financer toutes ses extravagances, les ressources du poète s'épuisent très vite. Un dernier recueil, synthèse des recherches poétiques de Sinadino - et quatrième volet de sa production - est publié à Milan en $1934^{10}$. Après la Deuxième Guerre mondiale et un séjour forcé dans un camp de réfugiés situé près de Bari, c'est à Milan que Sinadino finit sa vie, oublié de tous et ruiné, dans une modeste pension de Porta Genova. Lorsqu'il décède le 23 novembre 1956, les seules personnes qui prennent acte du «congé de l'Égyptien» (pour reprendre le titre de l'un de ses derniers poèmes ${ }^{11}$ ) font partie de sa famille d'adoption: la petite communauté des clients de la pension ${ }^{12}$.

Cet aperçu biographique, pure énumération de lieux de passage, constitue un ensemble difficilement intelligible. La difficulté provient d'une carence de données documentaires, renforcée, a contrario, par l'impression d'une vie dont la densité est excessive: la biographie de Sinadino contient une histoire très longue - elle s'étend sur une durée de quatre-vingts ans, de 1876 à 1956 -, très segmentée - elle est particulièrement riche en ruptures, déviations, coups de théâtre, abandons, départs et retours -, et très enchevêtrée - l'itinéraire parcouru par Sinadino passe (et repasse) par au moins quatre pays, liés à des séjours plus ou moins longs. Les coupures abondent, en somme, de tous points de vue, et empêchent de considérer ce parcours sans se perdre dans une vaine accumulation de lieux et de dates. Sinadino semble avoir réalisé le programme de l'bomme labyrinthique évoqué par Blanchot,opposé à l'bomme de mesure, dont l'espace est toujours borné: «pour l'homme mesuré et de mesure, la chambre, le désert et le monde sont des lieux strictement déterminés. Pour l'homme désertique et labyrinthique, voué à l'erreur d'une démarche nécessairement un peu plus longue que sa vie, le même espace sera vraiment infini, même s'il sait qu'il ne l'est pas et d'autant plus qu'il le saura» ${ }^{13}$.

La situation est identique si l'on considère la production du poète: l'œuvre de Sinadino apparaît atomisée, tant pour ce qui est des lieux de publication (Alexandrie, Milan, Lugano, Paris, New York) que pour ce qui est des supports (plaquettes à compte d'auteur, éditions courantes, revues littéraires), des genres de référence (poème, prose, théâtre), ou des modèles revendiqués (Novalis, Baudelaire, Poe, D’Annunzio, le symbolisme mallarméen, les proses philosophiques de Paul Valéry, l'hermétisme italien). Le parallélisme est évident: au déplacement perpétuel entre les

élégante plaquette constituée de fragments en prose (A. J. SinADINò, Il dio dell'attimo. Marginalia, Alessandria d'Egitto, Stabilimento A. Mourès, 1910); ce type d'écriture sous forme de «cahiers» sera repris bien des années plus tard, pour l'édition augmentée du volume (A. J. SinADINO, Il dio dell'attimo. Marginalia - Liriche - Varia. $I^{\circ}$ e $I I^{\circ}$ quaderno. Preceduti da una lettera di Paul Valéry, Milano, Bottega di Poesia, coll. «I Fascicoli di Bottega di Poesia», 1924). Ce sont des textes de ce type qui sont publiés dans la revue Grammata, entre 1917 et 1919.

(9) A. J. Sinadino, Poësies 1902-1925. I. - Cette fièvre appelée: vivre. II. - Cabier d'études spirituelles, Paris, À la jeune Parque, Marcel Sénac éditeur, 1929.

(10) L'ouvrage, publié par Enrico Dall'Oglio, s'intitule Vitae Subliminalis Aenigmata (A. J. SINA-
DINO, Vitae Subliminalis Aenigmata. Idillio d'Hyla, Milano, Edizioni Corbaccio, 1934).

(11) «Commiato dell'Egiziaco» est le poème qui clôt le recueil Vitae Subliminalis Aenigmata.

(12) La plupart des informations compilées dans ce paragraphe sont le fruit de recherches personnelles; elles prolongent les travaux du "pionnier" Ernesto Citro, dont l'essai consacré à Sinadino (E. Citro, Agostino Jobn Sinadino e la poetica del simbolismo, ravenna, Longo, 1986) contient, outre de précises analyses littéraires, quelques informations d'ordre historique et documentaire, glanées auprès des derniers témoins directs de la vie du poète, Joachim e Alessandra Esposito (Cf. en particulier pp. 137-141).

(13) M. Blanchot, «La question littéraire», in Le livre à venir, Paris, Gallimard, 1959, p. 116. 
pays et les langues correspond un autre type de passage, littéraire cette fois. Entre l'imprégnation et l'expérimentation, Sinadino garde une trace, dans son écriture même, de ses divers lieux de séjour littéraires. De ce fait, à force de rassembler - pour les refondre ensuite - les éléments les plus disparates de son expérience de lecteur, ce touche-à-tout génial contribue en profondeur au renouvellement des formes fixes du premier symbolisme, à leur transition vers les nouvelles configurations de la modernité. Malgré sa singularité, sa figure entre en résonance avec ses contemporains les plus novateurs, et nourrit secrètement la double veine «métaphysique» de l'hermétisme et du surréalisme.

\section{Lignes de fuite}

Mais au-delà du constat général d'une influence réciproque de la vie et de l'œuvre - sous le signe commun de la dispersion -, les contours de l' «être-Sinadino» ${ }^{14}$ restent flous et indéterminés. Faute de pouvoir proposer en une narration suivie l'histoire de sa formation, de sa culture, de son milieu (c'est-à-dire faute de pouvoir reproduire les schémas pré-construits de l'«illusion biographique» ${ }^{15}$ ), le seul moyen de restituer sa cohérence à l'expérience de Sinadino est d'accomplir une double opération: 1) intégrer en premier lieu cette figure solitaire et inclassable dans une communauté littéraire, en identifiant ses points communs avec les autres auteurs de sa génération; il s'agit de développer autour de Sinadino une recherche de type cartographique, de le mettre au centre d'une série de constellations. 2) Reconnaître d'autre part un certain nombre de grands motifs qui semblent structurer son existence, tels le nomadisme, la solitude, le dépouillement, qui deviennent aussi principes d'écriture. La biographie de Sinadino ressemble à un tableau sans profondeur: il s'agit de recomposer à partir de ses éléments épars une fausse perspective fondée sur ces deux lignes - qui ne sont, bien sûr, que des lignes de fuite.

1) Au jeu des affinités littéraires, qui a aussi fonction de reconnaissance et de classement ${ }^{16}$, la «proximité» la plus sensible est celle que l'on observe entre Agostino J. Sinadino et le groupe des Alexandrins. Alexandrins italiens, d'abord: Marinetti, Ungaretti, Pea, constituent un trio d'auteurs marqués par leur naissance ou leur passage dans la ville, qu'il convient de regrouper dans un même paradigme littéraire ${ }^{17}$; il

(14) Pour reprendre le titre d'un article très approfondi de Gian Battista Nazzaro sur la poétique de Sinadino. Il s'agit dans ce texte, pour Nazzaro, de démontrer que l'obscurité du poète est un choix à la fois littéraire et existentiel. Dissoudre sa propre personne de chair, effacer son identité, confier sa mémoire à la substance immatérielle de la littérature: voilà selon Nazzaro le projet littéraire de Sinadino, qui inaugure une nouvelle condition du poète - en quelque sorte «clandestine» - par rapport au reste du «champ» littéraire: «Allo storiografo non rimane che registrare questi sparsi elementi raccolti, nei quali l'essere Sinadinò si perde come l'immagine vana su di uno specchio deformante». Cf. G. B. Nazzaro, «L'essere Sinadinò», in ES, II, 3, février-mars 1975, pp. 73-98.

(15) Dénoncée avec vigueur par Pierre Bourdieu, comme mythe de la linéarité et de la cohérence, fondé sur le «postulat du sens de l'existence racontée»; le sens est ici à entendre à la fois comme signification et comme direction (P. Bourdieu, «Lillusion biographique» in Actes de la recherche en Sciences Sociales, 62-63, mai 1986, pp. 69-72).

(16) Si l'on songe à la théorie des "airs de famille" développée par Ludwig Wittgenstein et appliquée au domaine des jeux. On pourrait également faire référence à une autre mise en œuvre du même principe: la bibliothèque d'Aby Warburg, dont le classement, en constante évolution, n'obéissait qu'au critère - toujours renouvelé - de la "proximité" thématique. Il s'agit bien pour nous, sur le modèle de cette bibliothèque, de "classer" Sinadino à côté d'autres auteurs, au nom d'associations plus ou moins libres.

(17) C'est ce que propose en particulier François Livi dans l'introduction à son édition de la correspondance de Ungaretti avec ses amis égyptiens: ce paradigme égyptien de la littérature italienne (Marinetti, Ungaretti, Pea... et Sinadino) est en quelque sorte parallèle au paradigme uruguayen 
faudrait leur adjoindre le nom de Sinadino, quatrième exemple de sensibilità italiana nata in Egitto. Si ces quatre figures proviennent de milieux différents, si chacune appartient à une couche sociale bien déterminée de cette société multiculturelle mais très stratifiée (Sinadino et Marinetti fréquentent les classes les plus aisées de la ville, Ungaretti et Pea sont issus d'un milieu beaucoup plus modeste), tous garderont néanmoins dans leur écriture en langue italienne une trace de ce passage: la trace de l'Orient dont ils portent «le signe ombilical pourtant ineffaçable» ${ }^{18}$, ou du moins celle du microcosme très particulier d'Alexandrie (caractérisé par le plurilinguisme, le cosmopolitisme et la liberté des mœurs). Au-delà de ces trois noms, Sinadino appartient au cercle plus large des écrivains d'Alexandrie, qu'ils soient originaires de la ville ou simplement ses hôtes provisoires: de Henri Thuile à Pierre Benoît, de Valentine de Saint-Point à Elian J. Finbert, de Constantin Zograffo à Edward Morgan Forster (et, un peu après, à Henry Miller et Laurence Durrell), ces auteurs ont participé, comme lui, au développement de ce que Robert Ilbert nomme «un modèle éphémère de con-

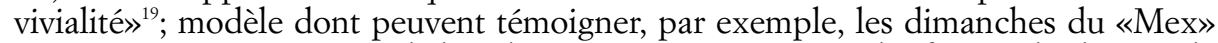
- où toute une aristocratie de la culture se réunissait autour des frères Thuile ${ }^{20}$ - et la revue Grammata dirigée par Stephanos Pargas, exemple d'exigence et d'ouverture intellectuelle, qui fédérait la plupart des initiatives littéraires. Au centre de cette galaxie se trouve la figure de Constantin Cavafis, âme de la ville, véritable étoile fixe dans l'ensemble mouvant - car sans cesse ravivé par de nouveaux arrivants - des lettrés d'Alexandrie. Si les rapports de Sinadino avec Cavafis ont sans doute été limités, une même approche poétique les rassemble, à tel point que Sinadino semble parfois l'inspirateur de certains textes du poète grec ${ }^{21}$.

Outre ce premier rapprochement, qui relève d'un déterminisme géographique commun, on peut identifier d'autres «airs de famille», entre le poète et les auteurs de

des lettres françaises (Lautréamont, Laforgue, Supervielle). Cf. à ce propos F. LIVI, Ungaretti, Pea e altri. Lettere agli amici egiziani, Napoli-Roma, Edizioni scientifiche italiane, 1988, p. 7. Le livre est une référence essentielle pour saisir le climat culturel d'Alexandrie au tournant du siècle - et pour compendre les divers réseaux de sociabilité qui se développent dans la ville.

(18) L'expression apparaît dans la lettre de Sinadino à Gide du 3 septembre 1927, écrite depuis Stresa.

(19) Robert Ilbert, par ailleurs auteur d'un ouvrage monumental sur Alexandrie (R. ILBERT, Alexandrie 1830-1930. Histoire d'une communauté citadine, Paris, I.F.A.O., 1996), évoque dans un ouvrage collectif justement intitulé Alexandrie 1860-1960. Un modèle éphémère de convivialité, Paris, Autrement, 1992, le développement puis l'échec, en l'espace d'un siècle, du modèle culturel d'Alexandrie, destiné à être balayé par la montée du nationalisme.

(20) Nous renvoyons encore une fois à l'ouvrage de F. Livi (Ungaretti, Pea e altri. Lettere agli amici egiziani, cit.) pour un tableau précis des après-midi du Mex, où se retrouvaient les principaux intellectuels de la ville. Deux lettres de Sinadino à Henri Thuile attestent - par leur tonalité générale - de sa présence régulière à ces réunions. Rappelons que Ungaretti a également fréquenté les frères Thuile entre 1911 et 1912, et que Pea a eu longtemps ses entrées au «Mex». On peut donc aisément imaginer une rencontre entre les deux Italiens et Sinadino - qui n'est pourant jamais cité par ces auteurs. Les raisons de ce silence, pour ne pas dire de cette réticence, restent mystérieuses. Ungaretti garde pourtant un souvenir très précis, à plus de cinquante ans d'intervalle, de la fabuleuse bibliothèque des frères Thuile: «ces amis [les frères Thuile] avaient hérité de leur père une magnifique bibliothèque, une bibliothèque romantique qu'ils avaient enrichie des poètes et des écrivains de leur temps, c'était une des plus belles bibliothèques privées que j'aie connues» (G. Ungaretti - J. Amrouche, Propos improvisés. Texte mis au point par Ph. Jacottet, Paris, Gallimard, 1972, pp. 30-31).

(21) Un texte comme La ville - sur le retour d'un voyageur dans sa ville natale - donne ainsi l'impression de dépeindre l'état d'âme de Sinadino à son retour à Alexandrie, en 1910 . Il ne s'agit bien sûr que de la convergence d'un même motif littéraire. Au-delà de ces parallélismes, remarquons que l'on trouve le nom d'un certain «Sinadino» dans un curieux brouillon de poème conservé dans les archives du romancier E. M. Forster, et dédié à Cavafis. Quand on sait par ailleurs que Cavafis fréquentait régulièrement A.Catraro, personnage très influent de la communauté italienne (c'est lui qui guidera Marinetti lors de son retour dans la ville, dont rend compte Il fascino dell'Egitto), on saisit mieux la superposition des diverses sphères de sociabilité d'Alexandrie - et on imagine les échanges transversaux qui pouvaient s'y développer. 
sa génération, liés à des choix littéraires spécifiques. Sinadino semble tout d'abord se placer de lui-même dans la constellation des immoralistes, dans la lignée de Wilde et de Gide(et de leurs doubles littéraires, Dorian Gray et Ménalque); ces auteurs sont des références constantes pour Sinadino, qui traduira en italien non seulement une des bibles de la littérature décadente, Salomé, mais aussi le chef d'œuvre de raffinement érotique qu'est Under the bill de Beardsley, l'illustrateur de la version anglaise de la pièce; en ce sens, Sinadino subit le magnétisme de modèles qui influencent, en Italie, toute une génération littéraire, comme le montrent la singulière convergence de traductions de Salomé au début du siècle ${ }^{22}$, ou l'afflux de lettres signées par de jeunes écrivains italien sur le bureau d'André Gide ${ }^{23}$; comme Wilde et Gide, du reste, Sinadino sera toujours considéré par les autres lettrés comme un irréductible excentrique, et nourrira autour de lui la rumeur de l'homosexualité.

Au nom de cette excentricité, Sinadino s'intègre dans un deuxième réseau, celui des esthètes et dilettantes, pour lesquels la vocation ne se réduit pas à la sphère littéraire, mais envahit d'autres disciplines, particulièrement la musique: Sinadino avait une réputation d'excellent pianiste, tout comme son ami Giuseppe Vannicola (18761915) passait pour un violoniste de grand talent. Leurs livres abondent d'analogies avec la musique, jugée reine des arts, sinon même «religion» ${ }^{24}$. L'attention que Sinadino porte à la réalisation graphique - et parfois aux illustrations - de ses ouvrages ${ }^{25}$ traduit également une sensibilité particulière pour les arts figuratifs, autre référence de son écriture. Pour Sinadino et Vannicola, l'esthétique développée dans les livres déborde donc de la bibliothèque et suscite d'autres formes d'expression; au-delà même de ces «correspondances» entre les arts, elle conditionne la vie tout entière, qui voudrait en être le dépassement, en tant qu'elle serait elle-même cette œuvre unique - drame ou roman, épopée ou tragédie - tant recherchée par les voies de la création $^{26}$. La vie considérée comme art total amène ainsi, naturellement, à des attitudes de dandysme et d'anticonformisme. Ce détachement des contraintes matérielles et cette recherche d'un vivere inimitabile rattachent Sinadino à la génération de la fin

(22) Trois traductions italiennes de Salomé, plus ou moins simultanées, ont été publiées au début du siècle: Salome: poema drammatico. Unica versione italiana consentita dall'autore, di G. G. Rocco, Napoli, F. Bideri, 1906; Salome: tragedia. Versione italiana curata da G. Vannicola, Roma, B. Lux, 1908; A. J. Sinadino, Salomé. Prima versione italiana, New York, Dante, 1909. Un procès a d'ailleurs opposé G. G. Rocco à G. Vannicola, au sujet de la traduction de Wilde (le premier accusant - à juste titre semble-t-il - le second de plagiat).

(23) Entre 1905 et 1910, Gide reçoit par exemple des missives signées Marinetti, Papini, Vannicola (et bien sûr Sinadino): une correspondance avec André Gide est à cette époque une nécessaire caution d'intellectualité. On trouve d'intéressantes indications sur ces contacts transalpins - d'autant plus soutenus qu'André Gide sera perçu comme une éminence grise de la N.R.F., pouvant faciliter l'accès à sa tribune - dans A. Goulet, Giovanni Papini juge de Gide, Lyon, Centre d'études gidiennes, 1982.

(24) De très nombreux aphorismes du recueil Il dio dell'attimo (1910-1924) évoquent la musique, non seulement comme forme expressive privilégiée («La Musica è anteriore a tutte le arti») mais aussi comme discipline révélatrice de la nature douloureuse de l'existence («La Musica è Dolore Puro. La condizione necessaria all'atto del Dio che crea») et comme intuition du divin ( $\ll$ Se il vento di Musica investa una piccola foglia: subito la fa senescere di luce, redenta; e la solleva, d'un grado, a più sottile sfera spirituale; più l'avvicina al tempo della sua risoluzion perfetta in Dio»). Cf. Il dio dell'attimo. Marginalia - Liriche - Varia, cit., «Teoria del «Dolore-Musica»», pp. 25-34.

(25) Sur la page de garde du "roman" La donna dagli specchi, publié à Milan en 1899, on trouve par exemple une superbe illustration du peintre symboliste Carlo Agazzi (1870-1922).

(26) C'est dans cette perspective que Ferdinando Gerra parlait du «drame de vie» de Giuseppe Vannicola, dans son remarquable essai sur l'existence du violoniste: F. GERRA, Musica, letteratura e mistica nel dramma di vita di Giuseppe Vannicola (1876-1915). La Revue du Nord e la rivista Prose, Roma, Bardi Editore, 1978. Signalons, à propos de Vannicola, le profil bio-bibliographique très documenté paru récemment dans la revue WUZ: A. Audoli, «Le prime di Vannicola», WUZ, I, 5, juin 2002, pp. 22-31. 
du siècle, avant la «cristallisation» du champ littéraire, avant que l'activité littéraire ne se constitue définitivement en métier.

C'est sans doute cet idéal qui entraîne Sinadino à (tenter de) construire son existence selon une succession de tableaux, et sa poésie comme une cascade d'instants. Cet aspect central de sa biographie - son «mouvement perpétuel»-le situe d'ailleurs dans la grande lignée des nomades sans attaches. La référence la plus immédiate est bien sûr Giuseppe Ungaretti, poeta-girovago par antonomase, d'autant plus proche de Sinadino qu'il provient également du creuset d'Alexandrie, et qu'il conçoit ses poèmes comme autant de stations dans un parcours; mais à bien y regarder l'itinéraire de Ungaretti est plus linéaire, son mouvement plus clairement orienté vers la recherche d'une patrie, d'une reconnaissance, d'une terre promise; le parcours de Sinadino - son aîné de douze ans - semble relever au contraire de la fuite, de l'égarement et de la dispersion: à la vie comme œuvre unique s'associe le modèle des Nourritures terrestres, de la liberté dans la dépossession - «Heureux [...] qui ne s'attache à rien sur la terre et promène une éternelle ferveur à travers les constantes mobilités ${ }^{27}-$ et, peut-être, le souvenir de Rimbaud: en ce sens, la fuite à New York de 1906 reproduit le célèbre adieu à la poésie du poète français ${ }^{28}$; mais elle correspond à un acte moins radical, puisque Sinadino recommencera à écrire dès son retour en Égypte. Dans la constellation des grands nomades, il serait peut-être plus fécond de rapprocher Sinadino de Blaise Cendrars (pour les livres annoncés puis abandonnés, pour les éditions rares et introuvables), ou du plus fortuné Valery Larbaud (pour l'atmosphère feutrée des grands hôtels, que fréquentait également Sinadino, et pour le bilinguisme); comme ces deux auteurs, Sinadino, en déplacement constant, semble n'avoir d'autre patrie que la poésie.

2) Parallèlement à ces effets d'échos et de résonance, on identifie dans les rares documents laissés par Sinadino (en particulier dans les éléments de sa correspondance) la présence d'un certain nombre de motifs, qui reviennent avec une fréquence obsédante. Cette articulation en grands pôles doit certes être considérée avec prudence pour ne pas sombrer dans un psychologisme arbitraire, mais elle donne à (entre)voir, sinon des «fragments de l'âme», au moins les constantes d'un rapport au monde. Il est certain, par exemple, que le bilinguisme, la naissance en équilibre instable entre les langues, dépassent l'accident biographique pour déterminer une existence, pour qualifier l'être-au-monde de Sinadino: Sinadino naît dans un espace multiculturel et dans une famille divisée entre les communautés; en ce sens, à force d'être entre les pays et entre les langues, il est sans origine; il n'y a pas de nostos pour ceux qui viennent de nulle part - et il ne peuvent éprouver qu'une nostalgie sans objet. Il est tentant de voir dans cette absence de points fixes, dans ce vide des attaches, la source d'une si longue errance: le déplacement perpétuel du poète est bien sûr motivé par des circonstances extérieures (en particulier son endettement chronique), mais il finit par apparaître comme un moteur de l'existence, comme une véritable détermination génétique. Pour preuve, lorsque Sinadino s'accorde des parenthèses de quasi-sédentarité, le voilà qui songe déjà à son prochain départ ${ }^{29}$, ou qui se perçoit comme pri-

(27) Pour reprendre l'un des préceptes de Ménalque. Cf. A. GIDE, Les Nourritures terrestres, in Romans. Récits et soties. Euvres lyriques, Paris, Gallimard, coll. «La Pléiade», 1958, p. 184.

(28) Comme le remarquait déjà G. B. Nazzaro, en évoquant ce chapitre de l'existence de Sinadino: «Poi rinuncia, sembra, anche alla letteratura, e passa il mare, per trasformarsi in qualcosa d'altro, commesso o impiegato di banca, perfettamente ingoiato dalla civiltà neocapitalista americana, come Rimbaud lo fu da quella colonialista europea» («L'essere Sinadinò», cit., p. 77).

(29) Comme le montre ce fragment du recueil Il dio dell'attimo daté «Ceccano (Roma) 1905», qui porte déjà sa propre vision nostalgique, au passé: «Quando il mio fervore inquieto m'avrà 
sonnier d'une terre hostile ${ }^{30}$. Le voyage devient exil, l'existence s'égrène en une série d'adieux: ce parti entraîne nécessairement une extrême solitude, que ne semble briser ni le mariage célébré en 1900 ni la correspondance entretenue avec divers auteurs. En ce sens, on pourrait aisément parler du parcours de Sinadino comme d'une série d'expériences du désert: du milieu littéraire italien du début du siècle auquel le poète ne s'intègre pas à l'exil de New York que Sinadino subit en vivant reclus dans son pavillon, du milieu parisien dans lequel Gide ne parvient pas à imposer son correspondant aux faubourgs de Milan de l'après-guerre, que Sinadino sillonne dans un état d'extrême précarité, le poète semble constamment entouré par le vide, fidèle à son image de «sensibile nomade, posto nel centro di barbarie varie» ${ }^{31}$. Si pour Rilke, selon la célèbre formule du Livre de la pauvreté et de la mort, chaque homme porte sa mort en soi, Sinadino au contraire porte son propre néantautour de lui. Le sable du désert non seulement l'entoure, mais recouvre ses traces.

On pourrait d'ailleurs se demander dans quelle mesure le poète ne contribue pas, volontairement, à cet effacement; la solitude ne semble pas subie, mais voulue et cultivée, comme preuve de malédiction et signe de supériorité - ce qui n'est pas sans rappeler le splendide isolement de Gian Pietro Lucini, souligné en son temps par Paolo Valera ${ }^{32}$. Significativement, dès sa première lettre à Gide, écrite depuis New York, Sinadino se présentera comme un poète vivant seul «parmi ces barbares», «en quête vainement d'un rayon, d'un accord, d'un parfum» ${ }^{33}$. Même dans les situations de plus grande intégration, Sinadino se trouvera dans une situation d'être aux marges, qu'il s'agisse de marges géographiques ou de marges sociales. Communauté familiale, monde littéraire italien, microcosme d'Alexandrie: orgueil ou crainte, Sinadino se placera régulièrement à la lisière des mondes qu'il fréquentera, il s'établira sur leur frontière extérieure sans jamais en pénétrer le cœur - à l'image du lac sur les bords duquel il a passé son adolescence. Une véritable force centrifuge, à propos de laquelle toutes les hypothèses sont possibles ${ }^{34}$.

trascinato lontano da questa calma terra d'esilio, sovente ripenserò a quell'angolo di muro tepente, ove, a' primi modulamenti della primavera, venivo a immergermi nel sole, dopo la colazione; ove lessi Amyntas di Gide, singhiozzante d'ebrezze ignote, sul vecchio sedile, nel commento del vento vasto e tra l'amoroso tubar de' colombi, vibranti nell'azzurro siccome frantumi di sole» (Il dio dell'attimo, cit., p. 56)

(30) Peu après son installation à Alexandrie - au retour de New York -, Sinadino écrit une lettre à Gide qui commence par ces mots: «De cette immobile terre, que toute ma tristesse contrarie et qui m'enchaîne» (lettre du 7 juin 1911).

(31) Pour reprendre une expression employée dans le «Préambule» au premier cahier du recueil Il dio dell'Attimo (Il dio dell'attimo, cit., p.13).

(32) Dans son article de 1914 sur Gian Pietro Lucini, Paolo Valera insistait en effet sur la solitude aristocratique du poète; ses remarques sur Lucini «uomo di lusso», situé hors du fracas de la vie moderne, peuvent aisément être appliquées à Sinadino, qui partage la même condition: «Lucini è morto. Io l'ho considerato intellettualmente un uomo di lusso. Scriveva quello che gli piaceva, come gli piaceva, quando lo sentiva. Era signore del suo ingegno. La sua produzione cerebrale, pur avendo in fondo il lievito rosso è rimasta aristocratica. [...] Ha disamato la réclame. L'ha creduta la fattucchiera dei ciarlatani. Non ha capito che è la tromba del nostro tempo. [...] È così rimasto fra noi come un uomo bizzarro che scriveva per gli ammiratori» (P. VALERA, «Gian Pietro Lucini è morto», in La Folla, III, 30, 26 juillet 1914 - reproduit sous le titre «La morte di Gian Pietro Lucini», in Il Caffé, 30 juin 1967).

(33) Lettre à Gide du 30 juillet 1909.

(34) C'est Ruggero Jacobbi qui est allé le plus loin dans l'interprétation de cette marginalité, en la rapportant à la marginalité par excellence, une probable tendance homosexuelle de Sinadino: «Sinadinò - a molti segni riscontrabili nei testi che possediamo - era certamente un omosessuale, od un bisessuale, secondo l'esempio del suo «concittadino»Kavafis e dei poeti arabi d'Egitto. E ciò gli dà una connotazione di «diverso», con una libertà morale molto cosmopolitica, che lo fece trovare particolarmente a suo agio - in Italia - solo a Firenze, nella Firenze raffinata della colonia inglese, e nei paraggi di Vannicola, con cui ha in comune diverse posizioni intellettuali ed anche il rapporto, quasi sicuramente personale, con Gide» (R. JACOBBI, «Agostino Sinadinò», in L'avventura del Novecento, Milano, Garzanti, 1983, p. 297). 


\section{L'attraction de la France}

L'existence de Sinadino prend en somme tous les aspects d'une traversée. Paradoxalement, cette traversée n'apparaît pas comme une recherche positive - quête d'une patrie, d'un ancrage, d'une situation sociale -, elle ressemble à une fuite et à une perte de soi. Les pays rencontrés par Sinadino ne semblent pas posséder à ses yeux d'attrait particulier, mais au contraire susciter déception et lassitude. «E me ne stacco sempre straniero» pourrait être l'un des mots d'ordre de Sinadino, qui se perçoit lui-même comme «étranger» perdu parmi la foule ${ }^{35}$. Une seule nation paraît conserver, un certain temps, une capacité d'attraction, et parvenir à modifier la trajectoire du poète, en l'attirant vers elle. Cette tendance fondamentale se dessine à partir du début du siècle et s'accentue vers 1910, date du retour des États-Unis. Il s'agit du lent rapprochement de Sinadino vers la France et la langue française.

De ce mouvement - rendu possible par une maîtrise linguistique presque sans faille - subsistent de nombreux indices: lectures très précoces en français ${ }^{36}$, composition de poèmes en langue française dès le début du siècle ${ }^{37}$, abonnement aux grandes revues parisiennes ${ }^{38}$, propositions de collaboration à la N.R.F. et à Commerce ${ }^{39}$, attention pour les nouveautés venues de France ${ }^{40}$, fréquentation de la communauté francophone d'Alexandrie ${ }^{41}$, publication d'un recueil en italien précédé d'une lettre-préface de Paul Valéry ${ }^{42}$ : dans leur accumulation, ces preuves dispersées révèlent la sympathie qu'éprouve Sinadino pour le monde littéraire français. Elles sont par ailleurs renforcées par une sorte de signe géographique, sensible à partir de 1920; Sinadino, alors

(35) Le thème de «l'étranger» apparaît à plusieurs reprises dans le recueil des Poësies 1902-1925 (en particulier dans «Poème moderne»).

(36) La correspondance et les premières œuvres de Sinadino contiennent par exemple des références à Paludes (1895), à l'Introduction à la méthode de Léonard de Vinci (1895), aux Nourritures terrestres (1897), ouvrages que le poète semble avoir lus, à chaque fois, dans leur première édition - témoignant ainsi de sa clairvoyance de lecteur, capable de prendre connaissance des ouvrages les plus marquants de son temps, avant même leur succès public.

(37) Les premiers poèmes que Sinadino compose en français sont probablement ceux qu'ils destine à la «Revue du Nord» de Vannicola, entre 1905 et 1907; mais on remarquera que le recueil des Pö̈sies publié en 1929 porte la mention «1902-1925», comme pour faire remonter le début de l'écriture en langue française à l'année 1902. Encore en aval, l'historien Jean-Jacques Luthi fait allusion, dans son ouvrage çonsacré à la littérature d'expression française en Égypte (J.-J. LuTHI, Introduction à la littérature d'expression française en Égypte. 1798 1945, Paris, Éditions de l'école, 1974), aux débuts d'un certain A. Sinadino dans une revue littéraire d'Alexandrie rédigée en français, Le Scarabée (vers les années 1895-1898). Quelle que soit la date retenue, on voit que le penchant français de Sinadino se manifeste précocement.

(38) Un indice dissimulé dans la correspondance Sinadino-Gide montre que le poète était abonné depuis Alexandrie à la N.R.F., tout comme il avait été abonné quelque temps auparavant à la revue de Paul Fort Vers et prose (cf. à ce propos la lettre du 7 juin 1911, et la note qui concerne Isabelle).
D'autres éléments dispersés dans ses textes tendent à indiquer qu'il lisait également Cosmopolis, Le Mercure de France, et Commerce.

(39) La lettre à Gide du 30 juillet 1909 se termine par exemple par la mention: «J'ose enclore des vers pour la N.R.F.»; celle du 3 septembre 1927 contient en post-scriptum: «Voici un fragment du petit poème qui a eu l'heur de vous plaire, et que la N.R.F. n'a pas cru devoir accepter». Une lettre à Paul Valéry, du 13 août 1928, commence par ces mots: «Cher Maître, je prends la liberté de vous soumettre pour Commerce deux poèmes: aspects ou profils qu'un même miroir a reflétés».

(40) Ainsi, dans la lettre à Gide du 7 juin 1911, Sinadino fait allusion aux représentations du Martyre de Saint Sébastien, qui ont lieu à Paris au même moment.

(41) Comme nous l'avons déjà indiqué, Sinadino a fréquenté avec assiduité le salon des frères Thuile; à tel point que Marie-Antoinette Michalla, seconde épouse d'Henri Thuile, gardait encore le souvenir de ses visites régulières, entre l'année de ses fiançailles (1919) et son départ définitif d'Égypte(1927). Cf. F. Livi, op. cit., note 4 p. 171.

(42) L'édition augmentée du recueil $\mathrm{Il}$ dio dell'attimo, publiée à Milan en 1924, est précédée d'une brève lettre de Paul Valéry, faisant office de préface: ce très beau texte - véritable poème en prose - est en réalité une lettre de remerciement envoyée au poète en 1923 (Sinadino avait offert à Valéry un exemplaire de son premier cahier); la correspondance retrouvée Sinadino-Valéry se réduit, à ce jour, à quatre éléments: entamée en 1922, elle témoigne d'un rapport d'estime et d'émulation, sans doute plus "professionnel" et moins intime que la relation Sinadino-Gide. 
établi en Égypte, multiplie les voyages en Europe, comme s'il manifestait le désir d'être plus proche de la France («de ce point, un peu plus rapproché de vous» écrira très significativement Sinadino à Gide en $1925^{43}$ ); mais ces indices disséminés ne sont, somme toute, que des éléments secondaires,qui entourent les deux documents essentiels de ce rapprochement: le premier est un recueil de poèmes en français, publié à compte d'auteur en 1929, dont la Bibliothèque Nationale de France conserve un exemplaire. Le second est constitué par la correspondance régulière que Sinadino a entretenue avec André Gide de 1909 à 1934, dont la plus grande partie, parvenue jusqu'à nous, a été exhumée par François Livi, puis complétée ponctuellement par Armando Audoli.

\section{Le recueil des Poësies: genèse, réception}

Le recueil intitulé sobrement Pöesies 1902-1925, publié à Paris en 1929, peut être considéré comme le couronnement, du point de vue éditorial, de cette rencontre de Sinadino avec la France. Cet ouvrage de la maturité - Sinadino a cinquante-trois ans au moment de sa parution -, qui dresse le bilan de vingt-cinq ans d'écriture en langue française, mêle les sources et les influences de façon très surprenante. Une partie de la correspondance Gide-Sinadino gravite autour de ce livre. Il n'est pas inutile, à ce titre, d'en proposer une présentation sommaire, en évoquant les circonstances un peu particulières de sa publication.

C'est André Gide, en contact épistolaire avec Sinadino depuis 1909, qui reçoit en 1927 le manuscrit des Poësies, expédié directement d'Alexandrie. Les deux auteurs s'étaient rencontrés à Paris quelques mois auparavant, en présence d'Alexandre Sinadino - frère du poète - et du jeune Marc Allégret ${ }^{44}$; l'envoi du manuscrit, à la fois preuve de confiance et signe d'intimité, marque le point culminant d'un rapport amical entamé presque vingt ans auparavant: «Je sais mal faire la part de l'affection que je vous porte, dans le plaisir que j'ai pris à vos poèmes, et je me parais à mes propres yeux bien suspect, car ceux qui me sont dédiés me touchent tout particulièrement. Je crois pourtant que je leur préfère encore ceux en vers irréguliers» déclare Gide à Sinadino le 18 novembre 1927. Prié de bien vouloir indiquer un éditeur auquel Sinadino pourrait soumettre le projet de recueil, Gide accepte de proposer quelques noms, mais - suprême coquetterie - fait mine de ne pas vouloir se séparer du manuscrit pour le transmettre à un éventuel lecteur: «Une chose me gêne: votre manuscrit est trop beau pour que je puisse consentir à m'en séparer, ou même simplement à le confier pour quelques temps à des mains étrangères. Ne disposeriez-vous pas d'une dactylographie que je pourrais montrer et prêter sans trop de crainte de l'abîmer ou

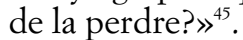

Cette lettre révèle assez bien, au-delà de l'embarras de Gide à juger la qualité du recueil, le rapport de séduction à distance qu'établit le don personnel du manuscrit,

(43) Ce sont les mots qui ouvrent la carte postale envoyée à Gide le 25 août 1925, depuis Stresa. C'est nous qui soulignons.

(44) La rencontre tant désirée par Sinadino a lieu entre mai 1926 (date du retour en France de Gide, après un long voyage en Afrique équatoriale) et septembre 1927 (date d'une lettre de Sinadino à Gide qui évoque le souvenir de cette rencontre, apparemment assez récent). Cf. à ce propos la lettre de Sinadino à Gide du 3 septembre 1927, et la lettre de Gide à Sinadino du 18 novembre 1927.

(45) Lettre du 18 novembre 1927 , retrouvée récemment par le critique Armando Audoli, dont une traduction italienne a été publiée dans la revue WUZ. Cf. pour plus de détails sur cette redécouverte la note 1 , à la suite de la transcription de la carte postale du 3 juillet 1923 . 
dont plusieurs poèmes sont dédiés à Gide ${ }^{46}$. Le manuscrit en question, aujourd'hui égaré, s'organise en deux cahiers, «Cette fièvre appelée: vivre» et «Cahier d'études spirituelles». L'ensemble esquisse un itinéraire symbolique du poète assez nettement balisé, à travers les deux états fondamentaux de la «fièvre» et de l'«étude». Mais dans le même temps, du fait de la présence massive d'éléments paratextuels parfois contradictoires - épigraphes et dédicaces, mentions de lieux et de dates, titres et sous-titres - il dessine aussi un labyrinthe, un réseau d'allusions et de références parfois très elliptiques, sinon tout à fait obscures. Ce sont les deux aspects fondamentaux du livre: l'un dynamique, linéaire, narratif, l'autre ramifié, réticulaire, enchevêtré, à l'image de l'existence même de Sinadino.

Comme l'indiquent les lettres suivantes, Gide prend à cœur de faire publier le recueil en France. Après quelques tâtonnements, un éditeur parisien est trouvé: ce sera Marcel Sénac, que Gide en personne se charge de contacter. L'ouvrage est imprimé en 1929, à compte d'auteur, dans une édition luxueuse, mais avec un tirage monochrome - contrairement à d'autres ouvrages de Sinadino, beaucoup plus inventifs du point de vuetypographique ${ }^{47}$. Les 311 exemplaires de la première impression sont destinés à une double distribution, à la fois en France (par Marcel Sénac) et en Égypte (par la prestigieuse maison Grammata ${ }^{48}$ ). Mais le projet se révèle trop ambitieux pour les finances de Sinadino, qui se trouve dans l'incapacité de rembourser l'éditeur; le livre est publié et distribué, mais les retards dans son financement s'accumulent, et Marcel Sénac finit par s'impatienter: un certain nombre de lettres de Sénac à Gide témoignent des réserves de l'éditeur devant les tergiversations de «l'italien» ${ }^{49}$.

(46) Le manuscrit est offrande, tout comme beaucoup des poèmes qu'il contient relèvent du don, car ils sont eux-mêmes dédicacés: «Tombeau et résurrection de Baudelaire» et tous les textes de la section «Profonde chair» sont dédiés «à André Gide» (le premier cahier du Dio dell'attimo portait aussi la mention «A | André Gide I «inquieto della diversità»»); on trouve également, dans le recueil, des textes dédiés «à Jean Cocteau», «à Paul Valéry», «à Henri de Régnier», «à Giorgio de Chirico», «à Pierre Benoit» - et encore «à la très blonde», «à mon frère Alexandre», «à Madame D. Z.»... Si l'on ajoute à cette présence massive de dédicaces les poèmes qui prennent la forme de «tombeaux» symboliques - «Tombeau et résurrection de Baudelaire», «Poe», «Novalis», «Whitman» - on obtient le panorama de ce que l'on pourrait baptiser une communauté d'élection: société fictive d'auteurs, ou d'amis, réunie par Sinadino autour du recueil parce qu'elle a «intelletto di poesia»: ce sont là, en quelque sorte, ses seuls compatriotes.

(47) Poësies 1902-1925, 205 x $140 \mathrm{~mm}$, couverture bleue. $141 \mathrm{p}$. Nom de l'auteur mentionné en couverture: Agostino J. Sinadino. Tiré à 311 exemplaires par Marcel Sénac, de la façon suivante: cinq exemplaires sur Japon impérial (numérotés de 1 à 5), soixante et un sur Lafuma (dont cinquante numérotés de 6 à 55 et onze numérotés de I à XI), et deux cent quarante-cinq correspondant à l'édition courante (numérotés de 56 à 300). Par rapport aux premières publications de Sinadino, l'édition du recueil est somme toute assez sobre: Le Presenze invisibili (1898), d'un format carré très inhabituel, était par exemple imprimé avec une encre vert émeraude; Melodie (1900), dont la couverture était violette, utilisait une encre rouge pour certains titres, sous-titres et lettrines; et surtout La festa (1900) déployait les mots sur la page sans respecter la typographie traditionnelle.

(48) Il reste une trace matérielle de cette double distribution: le recueil possédait deux couvertures légèrement différentes en fonction de sa destination géographique - sans que cette variation recoupe $a$ priori les distinctions présentées par la justification de tirage. La première couverture porte en bas de page la simple mention «A LA JEUNE PARQUE । MARCEL SÉNAC, Editeur | 3, place de l'Odéon | $P A$ $R I S \gg$. La seconde porte également cette indication, mais dans une police plus réduite, et laisse lire à sa droite, au même niveau: «MAISON D'ÉDITIONS | GRAMMATA | 6, rue de l'Église Debbanc | ALEXANDRIE (Égypte)».

(49) Par exemple, pendant l'été 1929: «Je n’userai pas du N. B., démocratisé par les femmes, pour vous dire la fin de l'histoire Sinadino. Il était convenu par contrat qu'il réglerait la seconde partie de sa note dès parution de son livre. Il m'écrivit pour me demander, contrairement à ce qui était stipulé, un mois supplémentaire de délai pour s'acquitter. J'eus la naïveté de lui envoyer ses livres et voilà trois mois que je suis sans nouvelles ni argent. Il ne répond plus. C'est tout.» (lettre de Sénac à Gide sans date, mais probablement de la fin du mois d'août); puis, en septembre 1929: «Il manquait à Monsieur Sinadino quelques volumes encore et voici qu'il me demande un nouveau délai et me prie de lui envoyer auparavant ces volumes. Dois-je le faire? Dans trois mois ce sera pareil je le crains et je serai trop loin pour le relancer. Que me conseillez-vous? Je m'en remets entièrement à vous» (lettre du 23 septembre 1929). 
C'est encore une fois Gide qui intervient, pour prendre l'initiative élégante de rembourser tous les frais ${ }^{50}$.

Ces indications, en apparence anecdotiques, fixent avec précision non seulement le cadre socio-littéraire dans lequel s'inscrit le recueil - une édition luxueuse à un tirage très limité, auprès d'un éditeur proche de la N.R.F. -, mais aussi son horizon de lecture idéal - Sinadino destine son ouvrage aux quelques francophiles éclairés qui résident en Égypte, et à la micro-société des lettrés parisiens, dont Gide est pour lui le premier représentant. Ce livre relève en somme plus du don personnel, de l'hommage à un cercle réduit d'écrivains, que d'une volonté abstraite de diffusion et de notoriété. Au sommet de la hiérarchie des lecteurs se trouve André Gide, à la fois destinataire réel (c'est à lui qu'est envoyé le manuscrit) et lecteur modèle du recueil (c'est à sa culture d'homme de lettres que semble s'adresser Sinadino).

Pour ce qui est de la réception de l'ouvrage, il semble que dans les mois qui suivent sa publication elle ait été très limitée, à la fois en France et en Égypte. Il est très probable que les difficultés financières de Sinadino, et par ricochet celles de l'éditeur, à la limite de la faillite au moment de la publication, aient empêché une publicité correcte. La seule appréciation critique positive apparaît quelques mois plus tard dans le prestigieux Mercure de France, sous la plume d'André Fontainas ${ }^{51}$. La «déviation» par la France de Sinadino s'achèveainsi dans la plus grande discrétion: si le recueil des Poësies existe bel et bien, une série de circonstances extra-littéraires limitent son retentissement. Hors de la sphère privée des connaisseurs, hors du «Maître et ami» André Gide, le recueil est destiné à une vie résiduelle dans l'ombre des bibliothèques.

\section{Agostino J. Sinadino - André Gide: chronique d'une amitié}

La publication du livre des Poësies a pour condition et pour arrière-plan l'amitié de Sinadino et de Gide, dont témoigne l'ensemble de dix-sept pièces de correspondance présentées dans les pages suivantes - ensemble qui constitue la deuxième grande trace «archéologique» de la saison française du poète d'Alexandrie. C'est très probablement par une lettre écrite au début de l'année 1909, dans laquelle Sinadino demandait à recevoir un exemplaire de La porte étroite, que commence un rapport épistolaire qui s'étendra sur une durée de 25 ans. S'il manque cette première lettre, ainsi que la plupart des réponses d'André Gide, si parfois on déplore d'autres lacunes dans les missives rédigées par Sinadino, et si l'on peut même supposer que la correspondance s'est poursuivie après la dernière lettre retrouvée $e^{52}$, les éléments qui subsis-

(50) L'épisode apparaît dans la correspondance Gide-Sinadino (lettre du 26 septembre 1929 et télégramme du 31 décembre 1929).

(51) Voici le texte intégralde la note rédigée par Fontainas, que l'on trouve dans le Mercure de France du 15 mars 1930(p.634-635): «Déjà une longue liste d'ouvrages écrits en langue italienne arrête l'attention sur le nom de M. Agostino J. Sinadinò. De 1902 à 1925 il a composé un certain nombre, un nombre respectable de vers en langue française, ce sont ses Poësies qu'il classe en deux cahiers: Cette fièvre appelée: vivre et Cabier d'études spirituelles. Le grand mérite en provient d'une sincérité à peu près héroïque, une connaissance remarquable de la langue et des exigences du vers français, qu'il soit régulier ou polymorphe, une ardeur de pensée, une fraîcheur de sensations et de sentiment, pure et délicate, fervente toujours. À en croire sa dédicace manuscrite, ce livre contiendrait "sa première hésitation sur le mode française" [sic]. L'auteur peut se raffermir et chanter sans crainte, il chante français, il ne tient qu'à lui de chanter en français». On remarquera l'ambiguïté de la dernière phrase, qui n'est pas sans une certaine noirceur.

(52) Ces lacunes sont principalement dues à la dispersion des affaires personnelles du poète, qui n'a pu gérer (faute de moyens) ni sa bibliothèque,ni ses manuscrits, ni sa correspondance; après la Deuxième Guerre mondiale, l'ensemble a été vendu par Sinadino à des libraires et des bouquinistes - on imagine avec quel déchirement. C'est d'autant plus regrettable que de nombreux témoins parlent de ces lettres comme d'un trésor dont Sinadino était très orgueilleux; pour ne citer que l'un d'eux, Henri Thuile évoque par exemple «Sinadino, qu'estimaient pourtant beaucoup Gide et Valéry, et à qui 
tent permettent de dessiner, par-delà les ellipses et les espaces blancs, la chronique d'une amitié singulièrepar bien des aspects.

Singulière d'abord par son extension, temporelle et géographique: la correspondance s'étend sur un quart de siècle, et comprend des lettres postées depuis cinq villes, situées dans quatre pays, sur trois continents ${ }^{53}$. Preuve, s'il en fallait, que le déplacement est intimement intégré à l'existence de Sinadino, qu'il en est le quotidien, qu'il en fonde la vérité. Singulière également par son déséquilibre: comme dans certains romans épistolaires, la parole est monopolisée par un seul correspondant ${ }^{54}$; il reste principalement des lettres de Sinadino (12 lettres sur un ensemble de 17), qui contiennent autant d'interrogations, d'attentes, de remerciements, dont le lecteur ne peut que deviner les objets ou les causes. Singulière, en troisième lieu, par sa construction à distance: elle traduit une amitié vécue in absentia pendant presque vingt ans, avant une unique rencontre, advenue à Paris entre 1926 et 1927. La «poignée de main», ardemment souhaitée dès la première lettre par Sinadino, sera sans cesse empêchée, ou reportée. Comme si cette correspondance, née sous le signe de La Porte étroite, en reprenait ironiquement le schéma de sublimation par l'absence, qui conduit les deux personnages du roman à être constamment séparés ${ }^{55}$. Simple rendezvous manqué dans les premières lettres, lorsque Sinadino séjourne à Paris, l'événement devient en effet un mirage de plus en plus lointain, un rêve de moins en moins réalisable: «une mauvaise divinité, par trop vraiment ironique, il me semble, persiste avec minutie à éviter notre tant désirée rencontre», écrit Sinadino en 1909, après avoir laissé échapper la possibilité d'une entrevue. Les années passent, les occasions de se voir se font plus rares, plus difficiles à organiser; le poète doit se contenter d'un «frêle fil télépathique» avec son correspondant. Vient enfin la «rencontresi inattendue et si chère à [son] cœur» dont Sinadino fait part à Gide dans une lettre de 1927. Cette entrevue marque un avant et un après, elle est un point de référence constant pour Sinadino, presque l'an zéro d'un nouveau calendrier. En 1933 il écrira encore: «que d'événements tristes et douloureux après notre tardive et seule rencontredont le souvenir m'éblouit toujours» ${ }^{56}$.

Dernière singularité de ces lettres: elles traduisent une amitié secrète, confiée à des plis cachetés. Gide ne mentionne jamais Sinadino dans les écrits intimes publiés à ce jour. Son nom n'apparaît pas dans son volumineux Journal, alors que beaucoup de contemporains, même mineurs, y sont cités ${ }^{57}$, et aucun indice, dans ce texte pour-

ils adressèrent des lettres des plus flatteuses» (Henri Thuile, entrevue pour La France Égyptienne, 16 avril 1955).

(53) Deux lettres de Sinadino sont écrites de New York (1909), deux autres de Paris (1909), quatre de Milan (1909-1929-1933-1934), deux d'Alexandrie (1911-1918), et deux de Stresa (1925-1927). Quatre lettres de Gide sont postées depuis Paris, une carte postale depuis Saint-Martin-Vésubie. En revanche, la poste fait suivre une lettre de Sinadino (Stresa 1925), qui rejoint l'écrivain français à Brazzaville.

(54) On songe parfois, en la lisant, à certains romans par lettres dont le correspondant est toujours «hors du champ»; le texte se transforme alors en «série ininterrompue de lettres d'un héros unique et solitaire à un ami qui n'est autre qu'un fantôme, ou une simple boite aux lettres» (J. RoussET, Le roman par lettres, in Forme et signification, Paris, José Corti, 1962, p. 70).

(55) Rappelons que le narrateur de La Porte étroite et sa promise Alissa cessent brutalement de se voir, et vivent chacun de l'absence de l'autre.
(56) Cf. respectivement, pour ces trois citations, les lettres du 30 juillet 1909, du 3 septembre 1927, et du 3 janvier 1933 .

(57) Pour se limiter à un florilège anecdotique, on trouve dans le Journal de Gide des jugements souvent cinglants - sur Marinetti («directeur d'une revue de camelote artistique du nom de Poesia»), D'Annunzio ( «il est petit;de loin sa figure paraîtrait ordinaire ou déjà connue, tant sur lui rien n'est pour montrer en-dehors littérature ni génie»), $\mathrm{Pa}$ pini («un peu trop pétulant [...]. Trop complimenteur; mais semble tout de même penserune partie de ce qu'il dit»), Vannicola («sa face tendre de Pulcinello; sa manie, quand il paie, de garder pour lui le cuivre et de laisser en pourboire l'argent»), et de nombreux écrivains ou intellectuels italiens ayant croisé son chemin. En ce sens, le Journal est aussi une sorte d'encyclopédie de la réception italienne en France. Pour les citations qui précèdent, cf. A. GIDE, Journal 1887-1925, Paris, Gallimard, coll. «La Pléiade», 1996, respectivement p. 443, p. 206, p. 545 , p. 692). 
tant friand de petits événements, ne permet de déterminer quand leur rencontre a pu avoir lieu. Cette absence est naturellement ambivalente: indice apparemment négatif d'un manque de considération, elle pourrait néanmoins être renversée, et considérée comme une marque de discrétion - en hommage à l'étrange amitié de celui qui se fait appeler l'ami sans visage $e^{58}$. Réciproquement, Sinadino fait preuve de la même réserve: si lorsqu'il échange quelques lettres avec Paul Valéry - certainement plus proche de lui pour ce qui est des choix et des modèles littéraires -, il n'hésite pas à exhiber dans la préface à l'un de ses recueils une lettre de réponse du poète français ${ }^{59}$, son amitié pour Gide reste plus silencieuse, sinon plus pudique. Sa manifestation extérieure reste limitée à quelques dédicaces dissimulées dans ses ouvrages.

Cette correspondance éthérée n'est donc pas le témoignage d'une amitié qui aurait d'autres canaux d'expression, elle est le moyen mêmede la faire vivre et de l'alimenter: si l'on exclut la «tardive et seule rencontre» entre les deux hommes, les lettres sont le seul biais pour entretenir un rapport qui s'éteindrait au moment même où elles cesseraient. Comme dans les célèbres Lettres à un jeune poète - entre deux correspondants qui ne sont qu'un nom l'un pour l'autre ${ }^{60}-$, chacune des missives de Sinadino participe à l'édification d'un lien fragile, qui ne tient que par le fil de la parole. Cette amitié, sans cesse réactualisée par l'écriture, évolue d'ailleurs avec le temps, comme évolue la façon dont Sinadino s'adresse à André Gide: du «Cher Monsieur» initial, à la fois courtois et très formel, au superlatif «Très cher Monsieur Gide» des lettres suivantes, jusqu'au «Très cher ami», voire au «Cher grand ami» des lettres finales. La correspondance retrouvée s'articule ainsi en plusieurs moments, qui sont autant d'étapes dans le rapport Sinadino-Gide: l'espoir d'une rencontre et l'idée d'une «communauté des nomadismes» dominent les lettres écrites dans l'année 1909. Puis commencent les années égyptiennes de Sinadino, et le projet - vite abandonné - d'une visite de Gide à Alexandrie. Enfin vient la rencontre«inattendue», à Paris; après ce contact se dessine un nouveau rapport. L'échange de lettres apparaît à présent motivé par le désir de publier un recueil: il accompagne la réalisation du projet et les problèmes qui s'ensuivent. La correspondance se transforme bientôt en une série de lettres angoissées qui traduisent les difficultés économiques de Sinadino, sa résignation progressive, son absence de perspectives: «Quant à ce que fais? Je passe mon temps à écrire, de plus en plus éloigné des lettres et des littérateurs d'ici...» ${ }^{61}$.

Ces lettres laissent deviner en somme une relation très particulière, faite d'attention et d'estime, entre un écrivain reconnu et un poète solitaire: témoignage de ce qu'a pu être la République des lettres en un moment précis de l'histoire littéraire - et prolongement immatériel, dans la correspondance, de cette «communautéculturelle» sans frontières dont Alexandrie avait été l'un des berceaux. À la toujours neuve inquiétude de Sinadino ${ }^{62}$ répond la disponibilité (cette disponibilité célébrée par Ménal-

(58) L'expression apparaît dans la lettre à Gide du 20 février 1918.

(59) Il s'agit, comme nous l'avons déjà indiqué, de la lettre-préface qui ouvre l'édition augmentée du recueil Il dio dell'attimo (1924).

(60) Les lettres de Rilke au jeune Kappus obéissent en effet à un schéma semblable: en déplacement constant à travers l'Europe, Rilke ne verra jamais le visage de son correspondant. Celui-ci choisira par ailleurs de ne publier qu'une sélection des lettres de son maître, en refusant d'inclure ses propres missives. Par rapport à la correspondance Gide-Sinadino, la relation est en quelque sorte inversée, puisque le lecteur n'a plus accès aux interrogations et aux doutes du jeune poète, mais seulement aux réponses de l'aîné.

(61) C'est le triste constat de la lettre du 3 janvier 1933 - mais cet aveu est aussitôt dépassé par l'évocation de nombreux projets littéraires: comme si l'écriture, considérée en tant que discipline privée, devait continuer indépendamment des possibilités de publication.

(62) Évoquée dans la lettre du 3 janvier 1933: «je vous souhaite ardemment une toujours neuve inquiétude! Car sans cela vous ne sauriez vivre!» 
que dans les Nourritures terrestres ${ }^{63}$ ) d'André Gide, prêt à accueillir la parole de son interlocuteur en toute occasion. Preuve d'bospitalité intellectuelle, la correspondance Gide-Sinadino délimite un nouveau territoire: à la fois lieu d'asile, retraite et refuge, ce «continent de mots» est la seule patrie possible pour le nomade Sinadino.

Profitant de cette promotion symbolique dans l'espace littéraire, Sinadino se confie librement. Ses lettres permettent d'observer non seulement l'éclosion - et l'abandon - de ses projets, mais aussi les fragments d'une vie intime dont les poèmes ne livrent qu'une version extrêmement travaillée. Contrairement à Gide, dont les ouvrages relevant d'une écriture de la confidence (journaux, carnets, correspondances) sont presque aussi volumineux que l'œuvre de fiction, l'intériorité de Sinadino n'apparaît à nu (si tant est qu'elle puisse apparaître sous cette forme) dans aucun autre texte. Habituellement, Sinadino pratique une écriture fermée sur elle-même, à mi-chemin entre la prière et le monologue. Ici, on constate à chaque page un effort vers l'ouverture, le dialogue, l'échange de vues, d'autant plus frappante que dans l'état actuel - en l'absence des principales lettres de Gide - sa parole reste comme suspendue dans le vide. Ces appelssans réponse trahissent un véritable désir de communication et de partage, dont on ne peut que deviner les prolongements. Il en ressort, chez le lecteur accoutumé aux constructions cérébrales de Sinadino, ou au raffinement des récits d'André Gide, une impressionde grande humanité, qui fait le prix de ces quelques lettres.

Nous tenons à remercier, au terme de cet article, Madame Catherine Gide, pour l'intérêt qu'elle a porté à nos recherches et la courtoisie avec laquelle elle nous a autorisé à publier les lettresde Sinadino; nous remercions également Monsieur Yves Peyré, Directeur du fonds Jacques Doucet, et l'ensemble du personnel de la Bibliothèque Littéraire, qui ont facilité notre consultation quotidienne de la correspondanced'André Gide. Une expression de gratitude, enfin, pour Monsieur François Livi, qui, ayant repéré ces précieuses lettres, nous a invité à en rédiger la présentation.

PAUL-ANDRÉ CLAUDEL

(63) «Chaque nouveauté doit nous trouver tout entiers disponibles. [...] «Don du poète, me disaisje, tu es le don de perpétuelle rencontre» - et j'accueillais de toutes parts. Mon âme était l'auberge ouverte au carrefour: ce qui voulait entrer, entrait. Je me suis fait ductile, à l'amiable, disponible par tous mes sens, écouteur jusqu'à n'avoir plus une pensée personnelle, capteur de toute émotion en passage, et réaction si minime que je ne tenais plus rien pour mal plutôt que de protester devant rien» (A. GIDE, Les Nourritures terrestres, in Euvres complètes, cit., p. 185). 
Note

La disposition générale du texte sur la page - retraits, espaces, etc. - a été respectée autant que la typographie le permet.

La transcription de chaque lettre est complétée par une note en caractères italiques, qui en propose une description sommaire selon leschéma suivant: indication de l'origine (collection, fonds, cote); analyse du support (format, en-tête, enveloppe); mention - quand il y a lieu - des corrections de l'orthographe ou de la syntaxe apportées au texte des lettres; remarques ponctuelles sur les particularités du manuscrit.

D'autres notes à caractère explicatif et documentaire, numérotées et en caractères romains, apportent des compléments d'information sur le contexte d'écriture et la situation des deux correspondants.

Enfin, les signes conventionnels suivants ont pu être utilisés, en note ou dans le texte:

[romain] Les mots en romain placés entre crochets sont rayés dans le manuscrit.

[italique] Les mots en italique entre crochets sont des mots ou des commentaires ajoutés par nous.

italique Les mots en italique dans le texte sont soulignés par l'auteur dans le manuscrit, ou correspondent à des titres d'ouvrages ou de revues dont l'écriture a été normalisée.

// // Les mots placés entre doubles barres obliques sont des ajouts supralinéaires ou en marge.

I Une barre verticale signale un saut de ligne.

$<>\quad$ Les mots ou les lettres entre soufflets sont de lecture conjecturale. 


\title{
1
}

\section{Sinadino à Gide}

\author{
New York - XXX. VII [1909] ${ }^{1}$ \\ 85, Park St.
}

\section{Cher Monsieur et ami.}

C'est avec un sentiment de plénitude spirituelle que je vous adresse ces mots.

L'envoi exquis de votre œuvre ${ }^{2}$ est venu combler l'attente pathétique et triste où je vis - parmi ces barbares - en quête vainement d'un rayon, d'un accord, d'un parfum ${ }^{3}$.

Aussi, voici que mes convictions esthétiques semblent s'accroître d'apports nouveaux.

Et je ne saurai considérer la vie - désormais - que comme une «contrainte belle» ${ }^{4}$ : aussi l'Art, «tout chemin qui mène à la perfection, à cette perfection «plus» celée» dont chante Moréas ${ }^{5}$, passe forcément par la Porte étroite. Une fois encore vos

1 Fonds Bibliothèque Littéraire Jacques Doucet (dorénavant B.L.J.D.), cote $\gamma$ 428-14. Lettre man. aut., sans env. Un seul feuillet grand format, $190 \times 285$ mm, plié en deux, écrit sur les quatre faces. Papier à en-tête: blason de la famille Sinadino, imprimé à l'encre rouge: aigle et heaume taré de profil, surmonté d'une couronne. Corrections orthographiques: celée en remplacement de: célée; forcément pour: forcement; éperdument pour: eperdument; vénusté pour: venusté. Correction de syntaxe: Urien pour: d'Urien. Autres remarques:une rature illisible: le levain [rature illisible] d'obscures angoisses immortelles; une correction manuscrite: vous plonge dans un[e barré] sentiment de «continuité contenue». Signature soulignée.

(1) Sinadino s'est établi à New York avec son épouse en 1906. Il quittera définitivement les États-Unis au début de l'année 1910. La datation de la lettre, ainsi que sa place inaugurale dans la correspondance retrouvée Gide-Sinadino, se déduisent de l'allusion à une œuvre dédicacée, qui est sans aucun doute possible La Porte étroite: le récit de Gide ayant été publié au Mercure de France en 1909 , cette lettre écrite depuis New York et citant La porte étroite remonte nécessairement au 30 juillet 1909

(2) Il s'agit, comme nous l'avons anticipé, du livre de Gide La Porte étroite, publié à 300 exemplaires sur Vergé d'Arches en 1909. Sinadino a très probablement demandé à Gide, dans une lettre antérieure (aujourd'hui égarée), de lui transmettre un exemplaire dédicacé de cette œuvre. Son ami Giuseppe Vannicola (1876-1915), violoniste de talent, écrivain dilettante et grand médiateur culture - dont le nom apparaîtra à plusieurs reprises dans les pages qui suivent -, mentionne d'ailleurs le livre, dans une missive à Gide du mois de septembre où il présente la même requête: «Vorrete farmi dono de La Porte étroite in volume? Sinadinò mi ha fatto vedere l'edizione - riuscitissima» (lettre du 3 septembre 1909conservée au fonds Jacques Doucet, cote $\gamma$ 839-1).

(3) Première apparition d'un thème obsessionnel de la correspondance de Sinadino, mais aussi de sa production poétique: l'isolement au milieu des «barbares», l'inaptitude à la vie moderne, dont les impératifs commerciaux entrent en contradiction avec la recherche poétique; on notera à ce titre la représentation de la recherche comme quête d'impressions - ou de correspondances - visuelles («un rayon»), musicales («un accord»), olfactives («un parfum»). Un poème écrit à New York dans ces années fait écho à cette affirmation: autoportrait de Sinadino en «étranger» perdu parmi la foule, cherchant «une étoile au-dessus des toits noirs» (A.J. SinAdino, «Poème moderne», in Poësies 19021925, Paris, Marcel Sénac, 1929, pp. 10-11).

(4) Expression que l'on retrouvera, attribuée à Gide, dans la lettre du 7 juin 1911 (lettre n 6), mais qui n'apparaît pas dans La Porte étroite; elle renvoie à une conviction maintes fois exprimée par André Gide («L'art naît de contrainte, vit de lutte, meurt de liberté»: De l'évolution du théâtre, 1904), mais aussi, plus globalement, à une koiné esthétique largement répandue au début du siècle (si l'on songe, par exemple, à Paul Valéry défenseur des «gênes exquises» de la composition poétique, dans Au sujet d'Adonis). Dans la lettre de Sinadino, le titre même de la Porte étroite se prêtera à la métaphore: l'art deviendra chemin de la difficulté, par un détournement singulier mais prévisible du récit gidien, interprété moins comme le récit d'une expérience vitale - la sublimation amoureuse - que comme une parabole esthétique.

(5) Allusion à un vers de Moréas, tiré du premier livre des Stances: «J'ai choisi cette rose au fond d'un vieux panier | Que portait par la rue une marchande rousse; I Ses pétales sont beaux du premier au dernier, I Sa pourpre vigoureuse en même temps est douce. II Vraiment d'une autre rose elle diffère moins | Que la lanterne fait d'une vessie enflée: | À ne s'y pas tromper qu'un sot mette ses soins, | Mais la perfection est chose plus celée» (cf. Jean MORÉAs, Les Stances, [1ère éd. 1899], in Euvres, Genève, Slatkine Reprints, 1977, vol. II, p. 18). À fois caution de littérarité (indice de culture), référence 
dons merveilleux se révèlent. La beauté de votre «composition organique» étonne et surprend tout délicat.

Et je rêve éperdument sous l'aile remuée de souvenirs: oh! ce Paludes ${ }^{6}$, le seul livre qui ait jeté dans ma trouble âme adolescente le levain d'obscures angoisses immortelles! Oh, la vision miraculeuse de cet «anneau» qui vous enserre, vous plonge dans un sentiment de «continuité contenue»... (Des sites délicieux du Lac Majeur en demeurent brumeux d'ivresse encore...)

Et mainte période nombreuse rit sûrement dans les plis de la mer d'Égypte: «sur tes flots, sur tes flots... mer éternelle» $\left(U_{r i e n}{ }^{7}\right)$. Et je divaguerais ainsi - sans fin.

Laissez-moi, donc, cher Maître et ami, vous décerner cette humble couronne de ma pauvre joie sans apprêt, et souhaiter avec vous l'avènement de cet «état nomade, sans attaches, sans possession $\gg^{8}$ auquel - rares - nous songeons parfois; soutenu comme je suis par l'espoir de bientôt vous serrer la main dans le cadre rêvé de quelque ville d'Italie où la lumière et la vénusté des choses puissent consentir, enfin, à la beauté du geste amical.

En toute cordialité votre

Agostino John Sinadinò

J'ose enclore des vers pour la N.R.F.'.

symboliste (signe d'une affinité théorique, même un peu datée), et modèle inavoué (image d'un lettré grec intégré à la France), la figure de Jean Moréas (1856-1910) remplit plusieurs fonctions, plus ou moins conscientes, dans les lettres de Sinadino. Jean Moréas, qui a donné sa charte au symbolisme (1886) avant de fonder l'École romane (1891), est d'ailleurs le seul nom de la sphère symboliste à être cité dans cette correspondance (son nom réapparaît dans la lettre $n^{\circ} 6$ ).

(6) Récit publié par Gide en 1895, à la Librairie de l'Art Indépendant - également cité par Sinadino dans son poème La festa (La festa, Lugano, TessinTouriste, 1901, p. 87), comme lecture apportant «infinito diletto». Le cadre du récit dans le récit (un étang isolé que parcourt jour après jour le personnage de Tityre) peut rappeler, ironiquement, l'atmosphère lacustre qui a marqué Sinadino pendant son enfance: la famille du poète possédait une luxueuse villa sur le Lac Majeur, à Stresa; mais c'est plutôt l'objet du récit - l'acceptation ou non d'une condition de confiné - qui semble en question dans l'appréciation de Sinadino.

(7) Citation approximative du Voyage d'Urien (1ère éd. 1893). En voici le texte exact, tiré de la première page du «Prélude», dans laquelle Urien s'abandonne à la rêverie avant d'embarquer: «Sur tes flots! Sur tes flots, pensai-je, voguerons-nous, mer éternelle, vers nos destinées inconnues? nos âmes excessivement jeunes chercheront-elles leur vaillance?» (Le Voyage d'Urien, in A. GIDE, Ro- mans, Paris, Gallimard, coll. «La Pléiade», 1988, p. 15). Récit d'une traversée imaginaire qui se change en quête métaphysique à travers les océans, le texte de Gide alimente le mythe du voyage-quête qui nourrit l'œuvre de Sinadino - et que le poète réalisera à la première personne, dans sa biographie. Cette citation du Voyage d'Urien esquisse une dialectique avec la précédente, tirée de Paludes, digne de L'Eau et les rêves: confinement et grands espaces, résignation et aventure, sédentarité et nomadisme, monde clos et univers infini - les deux pôles de l'existence de Sinadino -, trouvent leurs premiers symboles dans le lac et l'océan.

(8) Probable allusion à la dédicace manuscrite ajoutée par Gide à l'exemplaire de La Porte étroite - qui n'est pas sans rappeller certains «appels à la dépossession» présents dans les Nourritures terrestres.

(9) Après le «faux départ» de 1908 (un premier numéro de la revue, publié le 15 novembre, avait conduit les rédacteurs à revoir leur maquette), La Nouvelle Revue Française a pris son envol au début de l'année 1909 (avec la livraison du $1^{\text {er }}$ février, à laquelle est symboliquement assigné le $n^{\circ} 1$ ). C'est donc à une jeune revue que Sinadino transmet ses vers: mais la reconnaissance française ne viendra pas de la N.R.F., qui ne publiera aucun de ses textes. Cette surdité du monde des lettres parisien sera compensée par l'attention amicale de Gide, qui facilitera la publication, en 1929, d'un recueil de vers en français. 


\section{Sinadino à Gide}

[Paris, le] 27[-8-1909]

Cher Monsieur Gide,

En état de nomadisme de passage à Paris ${ }^{1}$, je vous adresse des vœux bien cordialement sincères avec le regret de devoir retarder encore le plaisir d'une entrevue.

Voici mon adresse à Milan

1, vicolo S. Margherita ${ }^{2}$

Bien à vous A. J. Sinadinò

\section{Sinadino à Gide}

[Milano, li] 3-9-[190]9

Très cher Monsieur Gide,

Las de voyages et d'émotions (une rencontre avec ma mère après 9 ans d'absence $^{1}$ et - particulièrement - le revoir d'un frère: Vannicola ${ }^{2}$ ) il m’est bien délicat

2 B.L.J.D., $\gamma$ 428-1. Carte post. man. aut., $90 \times 140$ mm. Date t. p. 27-8-09, Paris, Rue d'Amsterdam; 28-8-09, Paris, Rue de Rennes; 29-8-09, Criquetot l'Esneval, Seine Inférieur. Adr: André Gide Nouvelle Revue Française | 78, Rue d'Assas [barré] | Paris [barré]. Adr. corrigée: Cuverville | Criquetot l'Esneval (Seine Inférieur).

(1) Entre août et octobre 1909, Sinadino séjourne en Europe. Son voyage lui fait probablement emprunter une ligne maritime entre New York et la France: cela explique son bref passage à Paris, à l'aller (lettre $\mathrm{n}^{\circ} 2$ ) et au retour (lettre ${ }^{\circ} 4$ ) de Milan (d'où est envoyée la lettre $\mathrm{n}^{\circ} 3$ ).

(2) En termes de sociologie littéraire, il n'est pas inutile de remarquer que le vicolo $S$. Margherita, où séjournera Sinadino, est situé à quelques pas de la Scala et de la Galleria Vittorio Emanuele: cet étroit passage appartient à l'un des quartiers les plus élégants de Milan. Les autres adresses milanaises que fréquentera Sinadino lors de son séjour - la via Manzoni du Caffè Cova, la via S. Radegonda où est logé son ami Vannicola - esquissent une topographie de la bohème dorée, alternative à celle que parcourent les autres lettrés de la ville, si l'on songe par exemple au faste plus démonstratif de Marinetti, partagé entre son quartier général du corso Venezia et le restaurant Savini(la différence des lieux de sociabilité littéraire correspond à l'évidence à une différence de modèles et de conceptions esthétiques).

3 B.L.J.D., $\gamma$ 428-2. Lettre. man. aut., sans env. Un feuillet grand format, $220 \times 290 \mathrm{~mm}$, écrit recto verso. Papier à en-tête: première vignette: CAFFÉ Ristorante | Cova | Confetteria. Deuxième vignette: Cova. Au centre: Carlo Prati \& C. I Società per l'esercizio del I CAFFÉ-RISTORANTE E DELLA CON- fetteria | Milano | Via A. Manzoni, 1 - Piazza del Teatro alla Scala - Via G. Verdi, 2 | TelEFONO 8-13. Correctionde syntaxe: au Havre pour: à l'Havre. Remarques: une lecture conjecturate: toujours <altéré> d'une soif; une rature: pourrai-je vous voir [mot barré] à l'Havre?

(1) Après son mariage à Londres, en 1900, Sinadino a séjourné six ans à Ceccano puis trois à New York. Les neuf ans d'interruption dans les rapports mère-fils sont peut-être dus à un refroidissement après ce mariage soudain avec une femme de douze ans plus âgée, diversement apprécié par la famille de Sinadino.

(2) Giuseppe Vannicola, qualifié ici de «frère», est certainement la figure du monde littéraire italien dont la sensibilité présente le plus d'affinités avec celle de Sinadino. Rappelons que Vannicola a animé la vie littéraire florentine et romaine avec deux revues, la Revue du Nord (de 1904 à 1907), et Prose (1906-1908). Il a par ailleurs collaboré à Leonardo, L'Alba, Il Regno et La Voce, et publié plusieurs ouvrages, dont un roman, Sonata patetica. Sinadino a probablement rencontré Vannicola en 1905, à Florence ou à Rome. Ses collaborations à la Revue $d u$ Nord commencent peu après, et continueront jusqu'à la fin de l'aventure éditoriale de Vannicola, pour un total de dix textes publiés. La revue étant entièrement rédigée en français, les poèmes de Sinadino qui paraissent dans ces pages constituent ses premiers essais en langue française. Le lien entre les deux hommes - à la fois esthètes, poètes, et musiciens - ne cessera de se renforcer, jusqu'à la mort tragique de Vannicola, en 1915. Pour ce qui est des rapports entre Vannicola et Gide, nous savons que le rédacteur de le Revue $d u$ Nord appréciait particulièrement les œuvres de Gide, dont il a été le premier traducteur: en 1907, 
de vous écrire ces quelques mots, toujours altéré d'une soif, le désir de vous serrer la main. Je le souhaiterais durant ce bref séjour car pour la fin du mois je suis forcé de retourner en Amérique 3 .

Serez-vous à Paris pour la fin Septembre? Ou pourrai-je vous voir au Havre? ${ }^{4}$

Ce me serait une consolante lumière que d'emporter votre charmante présence là-bas et d'accroître ainsi mes ferveurs.

Avec Peppino5 bien sincèrement

1, vicolo S. Margherita

Milan

Agostino J. Sinadinò

\section{4 \\ Sinadino à Gide}

[Paris, le]19-X-[19]09

Aurai-je ce bonheur délicat de pouvoir enfin vous serrer la main avec la double «intention» de vous rapporter - tout frais - le souvenir et les salutations chaleureuses de notre Vannicola et de remporter pour ma part là-bas dans l'exil la précieuse vision d'une rencontre à laquelle je rêve si incessamment?

Je m'arrête ici jusqu'à vendredi soir ${ }^{1}$.

Hôtel Mirabeau - Chambre $114^{2}$.

il publie la traduction de La Ballade de la geôle de Reading d'Oscar Wilde, précédée du texte de Gide In memoriam, faisant office de préface (Oscar WIL$\mathrm{DE}$, La Ballata della prigione di Reading. Tradotta da Giuseppe Vannicola e preceduta dai ricordi di André Gide su Oscar Wilde, Roma, Bernardo Lux, 1907). La première rencontre entre les deux hommes, après un contact épistolaire établi en 1907, a lieu en mars-avril 1909, lorsque Gide passe six semaines à Rome: à en croire son journal, l'écrivain français est conquis par cet être «noué comme un cep» et «amoureux comme un pampre», en compagnie duquel il découvre «de Rome plus [qu'il n'a] jamais osé voir de Paris». Chargé par la N.R.F. de mener une véritable «chasse aux abonnés», Gide utilisera d'ailleurs les services de Vannicola - en concurrence avec Papini - pour diffuser la revue en Italie (cf. A. GIDE, Journal 1887-1925, Paris, Gallimard, coll. «La Pléiade», 1996, p. 692, et A. ANGLÈs, André Gide et le premier groupe de La Nouvelle Revue Française, Paris, Gallimard, coll. «Idées», 1978, vol I, La formation du groupe et les années d'apprentissage, $1890-1910$, p. 138).

(3) Le retour de Sinadino aux États-Unis ne durera que quelques mois: le temps d'organiser son départ définitif de New York. Sa présence est attestée à Alexandrie en 1910.

(4) Le, Havre: probable port d'embarquement pour les États-Unis.

(5) L'expression «avec Peppino» - «Peppino» étant le surnom familier de Giuseppe Vannicola - est à interpréter littéralement: les deux hommes se trouvent réellement côte à côte, à une table du fameux «caffè Cova» situé via Manzoni, près de la Scala... et chacun d'eux écrit une lettre à Gide: le fonds Doucet conserve en effet une lettre de Vannicola portant la même date (3 septembre 1909), et utilisant le même papier à en-tête du «caffè Cova». On trouve un deuxième témoignage de complicité entre Vannicola et Sinadino dans leur correspondance avec l'écrivain français: une petite carte postale envoyée depuis Milan, quelques jours plus tard, co-signée par Vannicola, Sinadino, Antongini - secrétaire particulier de D'Annunzio -, et un mystérieux «Paquet» - qui n'est autre... que le chien de Vannicola (carte postale conservée au fonds Jacques Doucet, cote $\gamma$ 839-5).

4 B.L.J.D., $\gamma$ 428-3. Lettre. man. aut., sans env. Un feuillet grand format, $210 \times 270 \mathrm{~mm}$, écrit recto verso. Papier à en-tête: Hôtel Mirabeau |8, RUE DE LA PaIX. Vignette: MirabEaU | ADRESSE TÉlÉGRAPHIQUE | Hôtel-MirabeaU-PARIS | TÉlÉPHONE. Corrections orthographiques: tout frais pour: tous frais; esprit pour: ésprit. Autre remarque: une rature: une rencontre [que] à laquelle je rêve.

(1) La lettre est du mardi qui précède (19 octobre).

(2) La mention de l'hôtel Mirabeau constitue - tout comme celle du Grand Hôtel des îles Borromées, dans la lettre $n^{\circ} 9-$ un indice sociologique intéressant: Sinadino fréquente, à Paris, l'un des fleurons de l'hôtellerie française, fondé au début du XIXe siècle, et situé rue de la Paix. 
Pourrai-je, cher M. Gide, promettre à mon esprit cette joie, à la sympathie que j'ai pour vous, cet apaisement?

J'attends.

Bien sincèrement

votre

A. J. Sinadinò

5

Sinadino à Gide

New York -3 Nov. [19]09

Cher Monsieur,

Une mauvaise divinité, par trop vraiment ironique, il me semble, persiste avec minutie à éviter notre tant désirée rencontre.

Voici que par un malencontreux hasard votre si aimable lettre me tombe entre les mains... en plein voyage ${ }^{1}$. Que de regrets... alors; que de tristesses...

J'aimerais tellement, qu'au moins, une subtile délicate amitié s'établissant entre nos deux «nomadismes» ${ }^{2}$, ces regrets, ces tristesses pussent doucement s'effacer.

Sur toute chose n'oublions jamais notre cher Vannicola. J'ai toujours dans la mémoire sa triste et étrange tête beethovénienne'. Qu'il me soit permis, cher Monsieur et Maître, de vous prier de quelque correspondance, de temps en temps...

Ces barbares me suffoquent tellement ${ }^{4}$.

5 B.L.J.D., $\gamma$ 428-4. Lettre. man. aut., sans env. Un feuillet grand format, $205 \times 255 \mathrm{~mm}$, plié en deux, écrit recto seulement. Papier libre. Corrections orthographiques: beethovénienne pour: beethovenienne; croyez-moi pour: croyez moi.

(1) La réponse de Gide à la lettre précédente de Sinadino, qui sollicitait une entrevue, lui est probablement parvenue au Havre, sinon à New York. D'après la teneur de ces lignes, on peut déduire que Gide y acceptait le rendez-vous tant espéré par Sinadino.

(2) Le «nomadisme» de Gide auquel Sinadino fait allusion - pour l'associer à sa propre expérience - tient sans doute plus d'une représentation filtrée par la lecture de ses œuvres que d'une connaissance biographique précise. Entre le nomadisme tempéré de Gide - avec ses deux pôles symboliques, l'Afrique du Nord et la France - et le nomadisme viscéral de Sinadino, incapable de se fixer plusieurs années dans le même lieu, il reste un abîme.

(3) Au-delà de la prédilection du violoniste Vannicola pour les sonates de Beethoven, et au-delà de sa ressemblance physique avec le compositeur, l'allusion à Beethoven renvoie à un tableau de Lionello Balestrieri: à Paris, en 1900, Balestrieri avait précisément profité - selon la légende - d'un moment où Vannicola interprétait la Sonate à Kreutzer pour le représenter en Beethoven, et peindre ainsi son tableau le plus célèbre (actuellement exposé au musée communal de Trieste).

(4) Sous la plume de Sinadino, l'appellatif «barbares», déjà employé dans la lettre $\mathrm{n}^{\circ} 1$, est l'expression d'un jugement sans partage, qui renvoie au substrat mythique du mot: ce peuple est «barbare» en ce qu'il est matérialiste et sans culture, mais aussi en ce qu'il ne peut produire qu'un langage fait de borborygmes, sons inarticulés loin des mélodies de la poésie (pour reprendre le titre d'un recueil de Sinadino). Le séjour du poète dans cette terre hostile, totalement cerné par les «barbares», représente donc un double exil - et un retournement du mythe de la terre d'accueil américaine. Remarquons que cette thématique de l'isolement dans un univers corrompu, dont la paternité peut être attribuée à Baudelaire, traverse la littérature décadente, au point de devenir un de ses lieux communs. Sinadino reprend d'ailleurs, pour qualifier sa situation, les mêmes éléments que Baudelaire évoquant la solitude américaine d'Edgar Poe: «les Etats-Unis ne furent pour Poe qu'une vaste prison qu'il parcourait avec l'agitation fiévreuse d'un être fait pour respirer dans un monde plus amoral, - qu'une grande barbarie éclairée au gaz -, et sa vie intérieure, spirituelle, de poète ou même d'ivrogne, n'était qu'un effort perpétuel pour échapper à l'influence de cette atmosphère antipathique» (C. BAUDELAIRE, Edgar Poe, sa vie et ses cuvres, in Euvres complètes, Paris, Gallimard, coll. «La Pléiade», 1976, vol. II, p. 297); ce mépris pour le matérialisme «barbare», véritable mot de passe d'une génération littéraire, se retrouvera, entre autres, dans le «Proemio» de la revue italienne Il Convito, dont Sinadino a probablement le souvenir; ce texte publié en janvier 1895 dans le premier numéro de la revue romaine - non signé, mais unanimement attribué à D'Annunzio - s'achevait par une exhortation à résister à la 
Excusez, donc, et croyez-moi bien sincèrement... inquiètement

85, Park St. New York.

Agostino J. Sinadinò

\section{6}

\section{Sinadino à Gide}

\section{Alexandrie, VII-VI-XI}

De cette immobile terre, que toute ma tristesse contrarie et qui m'enchaîne ${ }^{1}$, un salut, une élévation envers votre pensée, qui m'est coutumière, cher Monsieur Gide!

Puisque vous avez si bien défini l'Art: une «contrainte belle» que, du moins, je m'efforce de concentrer, au lieu de disperser mes ferveurs nichées dans tous les replis de ma sensibilité...

J'ai conçu une vaste «Tragoedia Deorum»² fruit de cette étrange angoisse, du conflit éternel entre les deux esprits: romantique-classique, une sorte de confrontation de tous les dieux avec le Christ, quoique - après tout - ce ne sont que bêtises, comme disait l'excellent Moréas à son lit de mort". L'essentiel est de «déformer» quelque point de vue, pour redonner les aspects que nous cache cette épaisse couche sensible!

«barbarie» au nom d'un esthétisme aristocratique («ciascuno di noi, pur da solo, secondo le sue forze, seguiterà a onorare e a difendere contro i Barbari i penati dello spirito latino»).

6 B.L.J.D., $\gamma$ 428-5. Lettre. man. aut., sans env. Un feuillet grand format, $200 \times 255 \mathrm{~mm}$, écrit recto verso. Papier libre. Corrections orthographiques: goûtée pour: goutée; télépathique pour: télepathique. Correction de syntaxe: Que pensez-vous pour: Qu'en pensez-vous. Autres remarques: un ajout supralinéaire: dans // tous // les replis de ma sensibilité. Signature soulignée.

(1) Au retour de New York, Sinadino s'est établi avec sa femme à Alexandrie - la ville de son enfance -, dans laquelle il demeurera dans les années 1910-1930. Le tableau de la terre d'Égypte, «cette immobile terre, que toute ma tristesse contrarie et qui m'enchaîne», est aussi négatif que celui de New York, mais sa caractérisation est différente: à l'activité frénétique succède l'immobilité paralysante, à la foule grouillante une prison de murs blancs. Le retour en Égypte est aussi un aveu d'échec. De ce point de vue, la proximité avec Constantin $\mathrm{Ca}$ vafis, autre prisonnier d'Alexandrie, cerné par les «barbares», est frappante. Sinadino semble faire sienne la prédiction fatale du poète grec, écrite en 1910: «Tu ne trouveras pas d'autres lieux, tu ne trouveras pas d'autres mers. [...] | Toujours à cette ville tu aboutiras. Et pour ailleurs - n'y compte pas - | il n'y a plus pour toi ni chemin ni navire» («La ville», in C. CAVAFIS, En attendant les barbares et autres poèmes, traduit du grec par D. Grandmont, Paris, Gallimard, coll. «Poésie», 2003, p. 53). L'air de famille entre les deux poètes d'Alexandrie ne se limite d'ailleurs pas à cette coïncidence ponctuelle: il englobe une culture, un milieu, une vocation, et une existence à la marge des institutions.

(2) De cette œuvre - également citée dans la liste des ouvrages «à paraître» du recueil $\mathrm{Il}$ dio dell'attimo de 1910 - il ne reste aucune trace: il s'agit probablement d'un manuscrit dont la publication, envisagée pendant un moment, a ensuite été abandonnée. Curieusement, le titre «Tragoedia deorum» apparaissait justement dans $\mathrm{Il}$ dio dell'attimo de 1910, pour distinguer une section du recueil. Quels que soient les interlocuteurs, les lettres de Sinadino évoquent souvent des œuvres à l'état de projet, dont le destin est bien incertain. De même, les listes des ouvrages «à paraître»ou «en préparation» présentes dans les recueils de Sinadino contiennent un très grand nombre de livres-fantômes, dont la publication est annoncée, puis reportée, enfin annulée. Ces ouvrages qui se réduisent finalement à un simple titre dessinent un espace très indéterminé: à la fois bibliothèque en pointillé, que le temps, la lassitude ou les contraintes financières ont empêché de réaliser et bibliothèque privée, résultat d'une écriture pour soi-même, dont la consultation est réservée au poète.

(3) Allusion à la confidence qu'aurait faite Jean Moréas à Maurice Barrès, quelques jours avant sa mort: «Il n'y a pas de classiques et de romantiques... C'est des bêtises...» (cf. M. Barrès, Adieu à Moréas [discours prononcé au cimetière du Père-Lachaise le 2 avril 1910], Paris, Emile-Paul, 1910, p. 7). 
Vous dire l'émotion «contenue» à la lecture de votre Isabelle $e^{4}$ que j'ai goûtée pour son objectivisme tout humain et son art...

Mais ne m'oubliez pas, cher Monsieur Gide, j'attends tellement quelque nouvelle belle chose de vous et... ne brisons pas ce frêle fil télépathique...

Cercle Mohammed Ali5.

Agostino J. Sinadinò

P. S. Que pensez-vous du Mystère de Mr. D’Annunzio ${ }^{6}$, n'est-ce pas le creuset où sont cuisinées des «planètes» et plus que des planètes?

\section{7 \\ Sinadino à Gide}

Alexandrie, 20 Février 1918

Cher Monsieur Gide,

La fréquentation de votre esprit m'est chère, mélancolique et chère, de tout ce que je connais, que je voudrais connaître et que j'ignore de vous.

Et s'il m'est permis, comme de la part d'un ami sans visage, de vous adresser un petit reproche pour le silence si prolongé de votre fine écriture que j'aime...

Et j'ai un grand remords aussi, que j'ose vous confier: d'avoir égaré (un des \# 3 sur Japon, le \# 1) des Nourritures ${ }^{1}$, que jamais, depuis lors, je ne voulus posséder dans l'édition courante.

Ce grand regret me pousse à une plus grande indiscrétion: vous demander 1 ex. de la nouvelle édition parue à la Nouvelle Revue Française (que je suppose épuisé),

(4) Ce «récit» (d'après le sous-titre de l'édition originale), d'abord publié par Gide en trois volets dans la N.R.F. (livraisons des 1er janvier, 1er février, 1er mars), est édité pour la première fois en juin 1911. L'achevé d'imprimer étant du 20 juin, c'est-à-dire postérieur à la date d'envoi de la lettre de Sinadino, on peut en déduire que Sinadino a lu Isabelle dans la N.R.F.: il est donc abonné à la revue depuis Alexandrie - tout comme il avait été abonné quelques années plus tôt, depuis Ceccano, à Vers et prose (cf. à ce propos le recensement des abonnés à la revue établi par F. Livi dans son article «Il teatro della morte. Corazzini e il simbolismo francese», in «Io non sono un poeta». Sergio Corazzini (18861907). Atti del convegno internazionale di studi. Roma, 11-13 marzo 1987, Roma-Nancy, Bulzoni editore, Presses Universitaires de Nancy, 1988, p. 168-169).

(5) Première mention du très élégant cercle Mohammed Aly, dont Sinadino est membre pendant tout son séjour à Alexandrie (son demi-frère, Michel Iannis Sinadinos, en est le président). Son papier à lettres caractéristique, marqué d'un sceau bleu, distingue à partir de cette date une grande part de la correspondance du poète.

(6) Allusion à l'actualité culturelle parisienne: il s'agit du Martyre de Saint Sébastien, dont les dix représentations se jouent précisément au moment où Sinadino écrit sa lettre à Gide (du 29 mai au 19 juin 1911).

7 B.L.J.D., $\gamma$ 428-6. Lettre. man. aut., sans env. Un feuillet grand format, $210 \times 265 \mathrm{~mm}$, plié en deux, écrit face 1 et 3, puis 2 dans le sens de la bauteur. Papier à en-tête: Sceau bleu: CERCLE MOHAMMED ALY. Remarque: deux ratures: votre [repris, trait plus fort] fugace impression sur [repris, trait plus fort] l'ami. Signature soulignée.

(1) Sinadino a donc possédé une véritable rareté, aujourd'hui très convoitée par les bibliophiles: le premier des trois exemplaires spéciaux, sur papier Japon, de la toute première édition des Nourritures terrestres (Paris, Le Mercure de France, 1897). Signe d'attention pour les grandes publications françaises, achetées avec discernement et... de gros moyens financiers, dès 1897 (Sinadino n'avait alors que vingt et un ans).

(2) La N.R.F. avait lancé une nouvelle édition des Nourritures terrestres, en 1917, de 300 exemplaires numérotés. Manifestement, la demande d'un ouvrage de cette série sert d'occasion ou de prétexte, de la part de l'«ami sans visage», pour rétablir un contact que l'éloignement menaçait de rompre. 
orné de quelque mot au charme toujours neuf pour dire votre fugace impression sur l'ami que vous n'avez pas connu et qui souffre toujours de ne pas vous connaître.

Cercle Mohammed Aly

Agostino J. Sinadino Alexandrie

8

Gide à Sinadino ${ }^{1}$

Hôtel Victoria, Saint Martin de Vésubie ${ }^{2}$

Stresa ${ }^{3}$ n'est peut-être pas bien loin d'ici... mais je n'ai ni cartes ni indicateurs. Tout de même j'aurais plaisir à vous rejoindre...

8 juillet [1923]

André Gide

\section{9}

Sinadino à Gide

Stresa ${ }^{1}, 25$-VIII-25

Villa Sinadino

Cher Monsieur et Ami,

De ce point, un peu plus rapproché de vous, je vous écris, très anxieux de connaître si vous êtes toujours disposé à agréer notre invitation (Les Amis de l'Art²) pour

8 Coll. part. Carte post. illustrée (St-Martin-Vésubie - Saison estivale - Vallée du Boréon I excursion à la Trinité), man. aut., $90 \times 140 \mathrm{~mm}$. Date t. p. illisible. Adr.: Sr Agostino Sinadino | Villa Sinadino | Stresa | (Novara) I Italie.

(1) Premier élément, dans notre ensemble de lettres, signé de la main d'André Gide. Cette petite carte postale a été retrouvée en juin 2005 par le critique Armando Audoli, qui avait également exhumé en 2004 les lettres n $11, n^{\circ} 12$ et n 16. Qu'il soit remercié pour sa courtoisie, sa disponibilité, et la facilité avec laquelle il a accepté de nous faire consulter les lettres originales.

(2) Gide a pris ses quartiers à l'hôtel Victoria, dans la station de montagne de Saint-Martin-Vésubie (Alpes Maritimes), en juillet 1923 (cf. A. GIDE, Journal 1887-1925, cit., pp. 1222-1226). C'est du reste la mention de ce lieu de séjour qui permet de dater la carte de Gide, dont le timbre postal est effacé.

(3) La famille Sinadino possédait à Stresa une villa, occupée depuis le début du siècle par le frère d'Agostino, Alessandro. Entre 1910 et 1930, le poète - établi à Alexandrie de l'automne au printemps - vient y séjourner presque chaque année, pendant les mois estivaux. Gide fait allusion à cette résidence parce qu'il se trouve dans les environs, mais se con- tente de caresser l'idée d'une visite: la tournure au conditionnel, le «j'aurais plaisirà vous rejoindre... »,diffèrent encore une fois la rencontre, et prolongent le jeu de cette correspondance aveugle.

9 B.L.J.D., y 428-7. Carte post. illustrée (S. Caterina del Sasso - Lago Maggiore), man. aut., $90 \times 135$ mm. Date t. p. 25-8-25, Stresa (2), 30-8-25, Paris, Rue de Rennes. Adr.: Monsieur | André Gide I clo Nouvelle Revue Française [barré] | Rue de Grenelle, 13 [barré] | Paris 2e [barré] | France [barré]. Adresse corrigée: clo Banque de l'Afrique Française | Brazzaville I France. Correction de syntaxe: disposé à agréer au lieu de: disposé d'agréer.

(1) Sinadino écrit à Gide depuis la villa de famille mentionnée dans la lettre précédente, dont il sera aussi question dans les lettres $n^{\circ} 10$ («je suis seul, ici, dans la villa de mon frère») et dans la lettre $\mathrm{n}^{\circ} 15$ («une villa à Stresa dont je viens de me défaire pour 235.000 lires»).

(2) En 1924, Sinadino participe, avec d'autres lettrés d'Alexandrie, à une association culturelle qui propose des lectures de textes et des conférences, «L'Atelier». Cette association - toujours active aujourd'hui - conservait une grande liberté d'action au sein du groupe plus élargi des «Amis de l'Art». Une lettre collective adressée à L'Égypte nouvelle, publiée dans son tirage du 13 août 1924, 
l'hiver prochain en Égypte. Dans le cas où vous pourriez, en principe, l'accepter, je vous saurai gré de bien vouloir m'en toucher un petit mot... Je vous rappelle que vous avez choix, depuis Nov. jusqu'à Février, du temps où vos loisirs vous permettraient de venir nous régaler de vos deux conférences promises ${ }^{3}$. C'est le seul moyen non déguisé qu'il me reste pour m'approcher enfin de vous. Mes salutations

Agostino J. Sinadino

10

Sinadino à Gide

\section{Villa Sinadino Stresa III-IX-XXVII}

Très cher ami,

Une carte, à tout hasard, expédiée, voici déjà une dizaine de jours, et dont le prix consistait en les quelques mots ajoutés par une incomparable amie': (Je salue André Gide invisible...). L'avez-vous reçue?

Je suis seul, ici, dans la villa de mon frère, qui n'est pas encor de retour. Après notre rencontre si inattendue et si chère à mon $\operatorname{cœur}^{2}$, mon dieu de l'instant ${ }^{3}$ me désigne tout un «corpus» de méditations, que j’avais par trop dédaignées.

documente la création de «l'Atelier» et permet d'avoir un aperçu des premiers membres. Parmi les signataires, outre Sinadino, apparaissent E. J. Finbert, D. Axisa, A. Saltiel, G. Petridès, B. Pascalis, E. Terni (cf. J.-J. LuTHI, Introduction à la littérature d'expression française en Égypte (1798-1945), Paris, Éditions de l'école, 1974, note 33 p. 90).

(3) Gide, parti pour un long voyage en Afrique équatoriale (de juillet 1925 à mai 1926), ne pourra se rendre en Égypte pour ces conférences. Rappelons que l'organisation de tournées de conférences pour les grands écrivains français constitue une initiative relativement répandue à l'époque. C'est précisément la même année que Giuseppe Ungaretti propose - vainement - à Henri Thuile d'inviter Paul Valéry en Égypte, en utilisant les services des «Amis de l'Art»: «Paul Valéry serait heureux de venir cet hiver en Égypte. Il ferait deux conférences à Alexandrie et deux au Caire. Je crois qu'il existe en Égypte une société qui s'occupe de ces choses. Naturellement, il faudrait donner un cachet à Valéry. Peut-être aimeriez-vous, d'accord avec la société en question, vous charger de l'organisation de cette tournée» (lettre à Henri Thuile du 27 avril 1925 , reproduite in F. LIVI, Ungaretti, Pea e altri. Lettere agli amici egiziani. Carteggi inediti con JeanLéon et Henri Thuile, Napoli, E.S.I., coll. «Inediti», 1988 , p. 123). On appréciera le parallélisme, sinon la concurrence, des deux projets: deux poètes tentent d'inviter sur leurs terres, en Égypte, deux écrivains français de premier plan avec lesquels ils entretiennent des rapports personnels. Au vu des dates, c'est peut-être la proposition de Giuseppe Ungaretti, relayée par Henri Thuile, qui a suggéré à Sinadino l'idée d'inviter Gide.
10 B.L.J.D., $\gamma$ 428-8. Lettre man. aut., sans env. Deux feuillets: premier feuillet grand format, $180 \times$ $340 \mathrm{~mm}$, plié en deux, écrit recto verso. Papier à entête: GRAND Hotel | É DES ILES BORROMÉES I STRESA, biffé de deux traits de plume. Deuxième feuillet petit format, $180 \times 170 \mathrm{~mm}$, écrit recto seulement. Correction orthographique: Tibet pour: Thibet. Correction de syntaxe: devenez-vous pour: devenez vous. Autres remarques: deux ajouts supralinéaires: le signe ombilical // pourtant // ineffaçable; un // fragment du // petit poème; une lecture conjecturale: une <Diotime $>$ du Nord. Signature soulignée.

(1) Il ne nous reste aucun élément sur cette «incomparable amie» dont parle Sinadino.

(2) Allusion à l'unique rencontre qui ait eu lieu entre les deux hommes: on peut la situer grossièrement entre mai 1926 (retour de Gide de son voyage en Afrique équatoriale) et septembre 1927 (date de cette lettre); elle a probablement eu lieu à Paris, en compagnie d'Alexandre Sinadino (cf. lettre $\mathrm{n}^{\circ} 14$ ) et de Marc Allégret (cf. lettre suivante).

(3) Cette expression - synthèse de la poétique de Sinadino - est la traduction littérale du titre d'un de ses ouvrages en italien: Il dio dell'attimo, Alessandria d'Egitto, A. Mourès, 1910; ce titre sera repris pour l'édition augmentée (Il dio dell'attimo. Primo e secondo quaderno, Milano, Bottega di Poesia, 1924), et reviendra même comme sous-titre dans le dernier recueil de Sinadino: Vitae Subliminalis Aenigmata. Il dio dell'attimo terzo quaderno, Milano, Edizioni Corbaccio, 1934. L'expression forgée par Sinadino, qui lie l'instant à l'éternel, le précaire au divin (ou mieux, qui intègre l'éternel dans l'instant, le précaire dans le divin, et laisse entrevoir une démultiplication des possibilités de 
Je me voulais «occidental» sans plus d'attaches avec l'Orient (dont nous portons le signe ombilical pourtant ineffaçable) et me voici replongé, grâce à cette amie (tellement contradictoire qu'on la dirait issue toute vivante de mes pensées les plus secrètes), dans l'étude des doctrines «yoga». Tibet...

Et cela, par l'exquise fréquentation d'une Diotime ${ }^{4} \mathrm{du}$ Nord qui revient du

Que devenez-vous, mon cher Gide? Que je serais heureux de recevoir un mot de vous... Je me sens si fier d'être considéré par vous parmi les «rares».

Et veuillez croire à mon affection particulièrement profonde.

Agostino J. Sinadino

Voici un fragment du petit poème qui eut l'heur de vous plaire, dans sa forme définitive, et que la N.R.F. n'a pas cru devoir accepter.

La perte de mes jours

voilés d'ailes étranges,

visités par mes anges

tutélaires, toujours

me remplit d'épouvante.

Ni la foule des fleurs

nouvelles que j'enfante

et j'ouvre aux profondeurs ${ }^{6}$

11

\section{Gide à Sinadino ${ }^{1}$}

Le 18 Novembre 1927

\section{Monsieur SINADINO \\ Cercle Mohammed Aly \\ ALEXANDRIE}

la conscience humaine), renvoie à une thématique post-nietzschéenne du surhomme qui traverse la production littéraire du début du siècle; pour ne citer que deux références - qui sont aussi les deux extrémités d'une parabole -, elle apparaît à la fois comme ambition démesurée dans Vita intima de Prezzolini (1903), et comme échec cuisant dans Un uomo finito de Papini (1913).

(4) Rappelons que c'est par la bouche de la «très savante Diotime», initiatrice de Socrate, qu'est révélée la nature secrète d'Amour, dans le Banquet.

(5) Probable allusion à une lettre - ou à une dédicace - de Gide.

(6) Comme dans la première lettre, Sinadino propose des vers à la lecture de Gide. On retrouve ce fragment, intégré dans le poème «Tel» du recueil Poësies 1902-1925, p. 119, avec quelques variantes de ponctuation et quelques modifications. En voici le texte complet: «La perte de mes jours | voilés d'ailes étranges, | visités par mes anges | tutélaires, toujours, II me remplit d'épouvante! | Ni la foule des fleurs | nouvelles que j'invente | et j'ouvre aux profondeurs, || ni l'or, les chevelures | qui traversent mes songes, I déchirant la froidure, I me sauvant des mensonges, || n'arrêteront du rire | les falotes phalanges! II D'autres voient un navire I qui s'avance plein d'anges...»

11 Coll. part. Lettre dact., sign. aut., sans env. Deux feuillets grand format, $210 \times 270 \mathrm{~mm}$. Papier à entête: inscription en rouge: 18 bis Ave des Sycomores 1 Villa Montmorency I Paris 16e. Correction orthographique: Mohammed Aly pour: Mohamed-Aly.

(1) Lettre dont la traduction en italien a été déjà publiée par la revue WUZ, publication spécialisée dans les livres rares et la bibliophilie (WUZ, III, 2, mars-avril 2004, p. 43). Comme nous l'avons déjà indiqué, nous devons au critique Armando Audoli, par ailleurs auteur d'un article consacré à Sinadino dans ce même numéro de la revue («Omaggio a Agostino John Sinadino, poeta amato e dimenticato», p. 40-46), d'avoir exhumé cette précieuse lettre de Gide, ainsi que les lettres $n^{\circ} 8, n^{\circ} 12$, et $\mathrm{n}^{\circ} 16$. 
Mon cher Sinadino,

Avec quel plaisir j'ai revu votre écriture et combien je vous sais gré de me rassurer si vite ainsi. Non certes, je ne trouverais aucune indiscrétion à ce que vous me demandiez ${ }^{2}$, si seulement j'étais certain de l'obtenir. Mais de récentes expériences me laissent croire qu'il n'y a pas grand espoir - du côté de la N.R.F. tout au moins, car elle est débordée (bien par sa faute du reste). S'il vous était indifférent de paraitre ailleurs, je pourrais essayer aux Librairies de la Porte Étroite ou de la Jeune Parque ${ }^{3}$.

Une chose me gêne: votre manuscrit est trop beau pour que je puisse consentir à m'en séparer, ou même simplement à le confier pour quelques temps à des mains étrangères. Ne disposeriez-vous pas d'une dactylographie que je pourrais montrer et prêter sans trop de crainte de l'abîmer ou de la perdre?

Je sais mal faire la part de l'affection que je vous porte, dans le plaisir que j'ai pris à vos poèmes, et je me parais à mes propres yeux bien suspect, car ceux qui me sont dédiés me touchent tout particulièrement. Je crois pourtant que je leur préfère encore ceux en vers irréguliers.

Marc Allégret ${ }^{4}$ me prie de vous dire qu'il est très sensible à votre souvenir. Il n'a pour le moment pas d'autre adresse que la mienne et c'est au 18bis Ave. des Sycomores que vous pouvez lui écrire. Je lui communique votre Post-Scriptum. Il se propose de vous écrire directement.

Veuillez croire à ma déjà vieille et fidèle affection,

André Gide

12

\section{Gide à Sinadino ${ }^{1}$}

Paris, le 7 Février 1928

Cher ami,

Un voyage en Allemagne ${ }^{2}$ avait inopinément arrêté mes démarches. Sitôt de retour de Berlin j'ai parlé à Marcel Sénac, nouveau libraire, à l'enseigne de La Jeune Parque, qui n'a guère édité jusqu'à présent que deux petits volumes de Valéry' (fort

(2) Sinadino a transmis à Gide le manuscrit d'un recueil de poèmes en français - qui deviendra deux ans plus tard Poësies 1902-1925 - en lui demandant un avis quant à la possibilité d'une publication. On observe dans les paragraphes suivants le rapport de séduction à distance qu'instaure le don de ce manuscrit, dont plusieurs poèmes sont dédiés à Gide («Tombeau et résurrection de Baudelaire», et tous les textes de la section «Profonde chair»). Comme en témoignent les lettres qui suivent, Gide prendra à cour de faire publier le livre en France.

(3) C'est effectivement à la Jeune Parque, dirigée par Marcel Sénac, que sera publié le recueil Poësies 1902-1925. Marcel Sénac était entré en contact avec André Gide à l'occasion de la parution d'Amyntas.

(4) Le jeune Marc Allégret (1900-1973), fils du pasteur Élie Allégret, et futur cinéaste, est à l'époque inséparable de Gide. Il était probablement présent lors de la rencontre entre Gide et Sinadino.

12 Coll. part. Lettre dact., sign. aut., sans env. Deux feuillets grand format, $210 \times 270 \mathrm{~mm}$. Papier libre.
Correction orthographique: Mobammed Aly pour: Mohamed Aby. Autres remarques: corrections manuscritesde l'auteur: [et] qui n'a guère édité; format, caractères, etc [et dessins].; [r]entré.

(1) Lettre dont la traduction italienne a été, comme la précédente, publiée par la revue WUZ (cit., p. 43).

(2) Gide s'est rendu à Berlin avec Marc Allégret, du 17 janvier au 3 février 1924 (cf. A. GIDE, Journal 1887-1925, cit., p. 70).

(3) Les deux volumes en question, dont l'édition est extrêmement raffinée, proposent chacun un bref texte de Paul Valéry: le premier (Durtal, ou les points d'une conversion, Paris, Marcel Sénac, 1927, tirage de 300 ex.), est agrémenté de bois et de lettrines de Thiollière; le second (...Huysmans, Paris, Marcel Sénac, 1927, tirage de 300 ex. également), qui reproduit l'article «Souvenir de J.-K. Huysmans» du recueil Variété II, contient une eauforte de Ch. Jouas. 
bien présentés), et qui me semblait particulièrement qualifié pour s'occuper de votre manuscrit. Longue conversation avec lui où il s'est montré plein de bonne volonté, mais désireux d'être rassuré au sujet de la question pécuniaire. Peut-être pourriezvous lui écrire directement (Marcel Sénac, la Jeune Parque, place de l'Odéon), afin de préciser ces quelques points:

I.-Aspects que vous souhaitez donner au volume (format, caractères, etc.). Le plus simple serait d'indiquer tel volume précédemment édité qui servirait de modèle.

II.-Tirage limité à combien?

III.-Vous parlez de souscripteurs? De toute manière, il faudrait que Sénac soit assuré de voir toutes ses dépenses couvertes. Je crois qu'il consentirait à ne pas tirer grand chose de cette édition, mais en tout cas devons-nous empêcher qu'il ne s'y échaude. Je crains même qu'il ne soit pas en état d'engager des fonds. Mais je n'ai pas pu lui dire quelles dispositions vous pensiez prendre à cet égard.

IV.-Point très important: votre dernière lettre parle de M. Émile Hazan, presque voisin de La Jeune Parque ${ }^{4}$. M. Sénac ne voudrait pour rien au monde entreprendre quoique ce soit qui pût causer quelque préjudice à un confrère avec qui il est en excellents termes. Si donc vous étiez déjà entré en rapport avec M. Hazan au sujet de ce projet, je crois que le mieux serait de continuer et d'abandonner Sénac, qui n'en prendrait aucun ombrage.

Je fais des vœux très affectueux pour la bonne réussite de votre livre. Tout heureux si je peux aider un peu à sa venue au monde.

Excusez cette dactylographie. De retour depuis deux jours j'ai trouvé une terrible accumulation de besognes de toutes sortes et n'ai pas le loisir de vous écrire comme je voudrais et comme mérite votre affection.

À vous bien sincèrement,

André Gide

\author{
Monsieur AGOSTINO J. SINADINO \\ Cercle Mohammed Aly \\ ALEXANDRIE \\ ÉGYPTE
}

13

\title{
Gide à Sinadino
}

Paris, le 26 septembre 1929

Mon cher Sinadino,

Je me suis permis d'envoyer de votre part à Marcel Sénac (qui vous en aura peut-être averti), 5.070 francs $^{1}$ (j'apprends du reste que la somme que vous lui devez

(4) L'éditeur de livres d'art et de volumes précieux Émile Hazan - dont l'entreprise connaîtra rapidement une certaine prospérité - s'était effectivement établi près de la place de l'Odéon $(8$, rue de Tournon).

13 B.L.J.D., $\gamma$ 428-13. Copie lettre dact. sans sign. Papier libre. Un feuillet grand format, $210 \times 270 \mathrm{~mm}$. Correction de syntaxe: moi-même pour: moi même.
(1) Par cette initiative élégante, Gide tente de combler les dettes que Sinadino a accumulées visà-vis de son éditeur Marcel Sénac, alors que le livre est déjà imprimé (l'achevé d'imprimer du recueil Poësies 1902-1925 est daté du 16 mai); un certain nombre de lettres de Sénac à Gide, conservées au fonds Jacques Doucet, témoignent de sa perplexité croissante devant l' «histoire Sinadino»: leur fré- 
encore est de mille francs plus élevée). Je vous en prie, ne voyez aucune indiscrétion dans ce geste, mais bien un désir de vous obliger en vous accordant ce nouveau délai que vous demandez, et d'obliger également Marcel Sénac qui doit quitter Bordeaux d'un jour à l'autre ${ }^{2}$, et ne peut par conséquent attendre davantage le règlement de ses factures. Excusez-moi si j'ai cru bon de me substituer à vous pour ce paiement, et à lui, par conséquent, vis-à-vis de vous, qui devrez désormais cette somme non plus à lui, mais à moi-même.

Veuillez croire à mes sentiments bien cordiaux.

quence augmente pendant l'été, à mesure que le retard et les dettes se creusent. À l'inverse, une lettre de Sinadino à Marcel Sénac, conservée parmi les documents de Gide (car transmise à l'écrivain par Sénac, soucieux de ne rien lui dissimuler), rend compte des difficultés financières du poète, et de son embarras face à l'éditeur:

«Monsieur Sénac,

Des malheurs successifs survenus l'un sur l'autre durant mon séjour chez mon frère à sa villa de Stresa, malheurs qu'il serait oiseux de vous narrer, car ce récit, j'en suis sûr, ne saurait dissiper vos doutes quant à sa sincérité, hélas, m’ont empêché d'être fidèle à la promesse que je vous avais faite (le retard du premier envoi des 3000 francs d'Égypte ne m'est redevable d'aucune façon, car cet argent je l'avais remis moi-même à M. Zelitas avant mon départ d'Alexandrie). À cela, en toute franchise [repris plus fort, par-dessus le mot sincérité], l'impression indéfinissable causée par votre lettre du 17 août 1929 à laquelle j'aurais tant voulu répondre par l'envoi pur et simple de la somme de $5070 \mathrm{frs}$, si la disponibilité de ce montant m'eut [sic] été laissée par ces malheurs imprévisibles!

Je ne tiens absolument [pas] à ce que vous souffriez de ce retard malheureux, car je vous suis on ne peut plus reconnaissant du zèle apporté à la fabrication de ce malheureux recueil, dont la haute valeur spirituelle, je dois bien l'avouer, pourra bien se faire jour, malgré une publicité manquée et prématurée... et je vous demande, comme suprême faveur, de m'accorder un délai de 3 mois, c'est[-]à[-]dire jusqu'à fin Décembre 1929 , date à laquelle vous voudrez bien me permettre d'ajouter au montant qui vous est dû la somme de 1000 francs, c'est[-]à[-] dire une totalité de 6000 francs.

D'ici là, je vous prie et je vous autorise de disposer de l'intégralité de l'édition, exception faite des 30 exemplaires que vous aurez l'obligeance de faire parvenir à Mons. Nicolas Zelitas Maison Grammata à Alexandrie (6 rue de l'Église Debbanc), ainsi que des exemplaires dédicacés à remettre aux destinataires.

Je vous prie, Monsieur Sénac, de bien vouloir donner des dispositions pour que la traite mise à mon nom et adresse soit provisoirement retirée, cela pour vous éviter des frais inutiles.

Je me sens bien fautif et accablé et ne sais comment invoquer votre indulgence et toute votre compréhension à mon égard.

Veuillez croire, cher Monsieur Sénac, à mes sentiments bien sincères de haute considération.

Agostino John Sinadino [souligné]

Jusqu'à la semaine prochaine à Stresa. Ensuite à Milan Vicolo S. Margherita N. 1».

(Lettre man. aut., sans env., datée du 20 septembre 1929, portant la mention «Monsieur Marcel Sénac | 197, Quai de Brazza | Bordeaux Bastide | (Gironde)». Trois feuillets grand format, bleu clair, deux recto-verso et un recto seulement. Papier libre). On remarquera que le recueil des Poësies devait aussi bénéficier d'une distribution en Égypte, par le biais de la prestigieuse «Maison Grammata» dirigée par Nicolas Zelitas (également rédacteur de la revue Grammata, sous le nom de Stephanos Pargas: ce même Stephanos Pargas, ami de Cavafis, auquel $\mathrm{H}$. Thuile avait dédié son ouvrage Littérature et orient quelques années plus tôt); le recueil de Sinadino conserve d'ailleurs une trace de cette double distribution, puisque certains exemplaires portent en couverture, à côté de la mention de la Jeune Parque, le nom de la «Maison d'édition Grammata».

(2) Marcel Sénac - grand voyageur - avait prévu de longue date de partir pour une navigation de plusieurs mois, de l'Atlantique vers l'Océan Pacifique, jusqu'en Océanie («je vous reverrai dans deux, trois, ou peut-être quatre ans», écrit Sénac à Gide le 16 septembre); mais ses projets, fixés pour l'automne 1929, sont bloqués par Sinadino, dont l'argent lui est nécessaire pour financer les derniers préparatifs: en ce sens, on pourrait dire que le nomadisme de l'un - perdu entre Alexandrie, Stresa et Milan - contraint l'autre à l'immobilité. Pour l'anecdote, signalons qu'une fois les problèmes financiers réglés, au moment du grand départ, l'équipage sans expérience de Sénac, pris de panique à quelques encablures de la côte, le contraindra à faire demi-tour et à renoncer définitivement à ses rêves de grandes traversées (réduisant ainsi, rétrospectivement, les torts de Sinadino). 
14

Sinadino à Gide

Milano 38804243116 [31-12-1929]

ÉMU GESTE AMICAL SILENCIEUX ANGOISSÉ JUSQUE MOMENT PROCHAIN CIRCONSTANCES PERMETTENT REMPLIR ENGAGEMENT DETTE HONNEUR ${ }^{1}$ MA PRÉSENCE AUGURALE = SINADINO

\section{5}

\section{Sinadino à Gide}

Milan, 3 janvier 1933

\section{Via Leopardi 21}

Cher grand ami,

Je ne saurais exprimer mon émotion le tremblement de ma main en reconnaissant ces volutes légères de votre écriture en lisant votre petite lettre ${ }^{1}$ qui me dit encor le souci affectueux que vous conservez de ma pauvre ombre malgré mon silence si long que vous devez certainement juger coupable et qui n'était qu'angoisse...

Que d'événements tristes et douloureux après notre tardive et seule rencontre ${ }^{2}$ dont le souvenir m'éblouit toujours. D'abord la perte de ma chère mère, il y a deux ans, en Septembre, et - après deux mois - celle plus cruelle encore de mon frère Alexandre $e^{3}$ que vous avez entrevu à Paris. Et, encor, à la suite de tout cela les démêlés horribles avec des conjoints riches à propos d'une succession, dont on a fini par me frustrer ne me laissant que charges et une villa à Stresa dont je viens de me défaire pour 235.000 lires somme qui constitue tout mon avoir que j'ai voulu investir dans une petite affaire d'immeubles de rapport au $10 \%$ garanti par mon associé pour la durée de 5 ans et sur laquelle je retire un mince revenu pour moi et ma femme.

Autre raison et - au fond - la seule de mon silence envers vous, mon admirable maître et ami - (car je n'aurais jamais pensé vous importuner par tous ces détails qui précèdent si votre amicale invitation ne m'y eût autorisé) la dette que j'ai contractée envers vous, lors de la publication de mes Poësies (que je juge bien inutile aujourd'hui).

14 B.L.J.D., $\gamma$ 428-9. Télégr. Date t. p.: 31-12-29, Paris Central; 31-12-29, Paris, Rue Poussin; 1-1-30, Paris, Rue de Grenelle. Adr.: Gide 18 bis Avenue [barré] | Sycomores [barré] Paris. Mention manuscrite: faire suivre 1bis Rue Vaneau.

(1) Cette «dette d'honneur» renvoie à l'évidence au geste de Gide évoqué dans la lettre précédente.

15 B.L.J.D., $\gamma$ 428-11. Lettre man. aut., sans env. Cinq feuillets petit format, $155 \times 235 \mathrm{~mm}$, numérotés, écrits recto verso. Corrections orthographiques: événements pour: evenements; démêlés pour: demêlés; ne m'y eût pour: n'y m'eut; contractée pour: contracté; déchirées pour: déchiré; indestructible pour indistructible. Autres remarques: ajouts supralinéaires: il y a deux ans, // en Septembre // et-après deux mois; que je juge bien inutile // aujourd'bui //; à vous faire parvenir, // à faire parvenir // jusqu'à votre cour; toute mon angoisse // qui // fond; mots barrés:je retire [une] un mince [rente] revenu. Signature soulignée.

(1) Allusion à une lettre aujourd'hui égarée de Gide à Sinadino.

(2) Confirmation du fait que Sinadino et Gide ne se sont rencontrés qu'une seule fois, à Paris, entre 1926 et 1927 (cf. encore, à ce propos, la lettre $\left.n^{\circ} 16\right)$.

(3) Margherita Carolina Casati, mère d'Alexandre et Agostino Sinadino, est décédée en 1930 - quarante ans après leur père, Ioannis Constantin Sinadino (1820-1890). Avec le décès presque contemporain d'Alexandre, c'est donc toute la famille proche de Sinadino qui disparaît, le laissant seul avec son épouse et un capital très limité, qui sera rapidement épuisé. 
Que de lettres explicatives torturantes et compliquées je vous ai écrites que j'ai déchirées ensuite, gardant sur ma conscience le poids de mon angoisse avec le sentiment d'impuissance à vous faire parvenir, à faire parvenir jusqu'à votre cœur immense et généreux le cri de mon admiration attentive et toujours étonnée, de mon affection indestructible!

Et, maintenant, par ce seul geste de votre lettre, par la musique de ces quelques mots, toute mon angoisse qui fond, remplacée par cette autre de nature plus délicatement inquiète qui me pousse à rattraper le long silence perdu!

Sur un mot de vous, me le permettant, comme je viendrais vous voir, ou si - d'ailleurs - le caprice vous souriait d'un petit séjour à Milan chez moi...

Je viens de recevoir le $1^{\text {er }}$ tome de vos œuvres complètes ${ }^{4}$, en cette édition adorable et par un étrange hasard j'étais en train d'en caresser la compacte épaisseur lorsque votre lettre m'a été remise.

Puis-je oser vous la faire parvenir pour que vous y inscriviez un mot d'affection, mon cher Gide?

Quant à ce que je fais? Je passe mon temps à écrire, de plus en plus éloigné des lettres et des littérateurs d'ici'... J'ai un volume de mémoires (mais objectivé en une sorte de roman «à la troisième personne») Il Fauno inginocchiato ${ }^{6}$ et j'achève en ce moment un recueil de poèmes en italien, Vitae subliminalis ÆEnigmata ${ }^{7} .$.

Mais laissez-moi vous apporter ici, comme la seule digne conclusion de cet écrit désordonné, l'expression de mon enthousiasme pour cette suprême manifestation de votre franchise - héroïque, qui vous permit de trancher d'un seul coup le nœud gordien de vos douloureuses inquiétudes contradictoires en proclamant très haut votre foi humaine et pure ${ }^{8}$.

Mais... à ce propos, permettez à l'ami de formuler ici un vœu... Me flattant de connaître quelque peu la nature merveilleuse de votre cœur épris de sincérité et ne désespérant de déchiffrer aussi celle - plus secrète - de votre esprit, je vous souhaite ardemment une toujours neuve inquiétude! ! car sans cela vous ne sauriez vivre!

(4) Il s'agit du tome I de l'édition des Euvres complètes d'André Gide, publiées à Paris à partir de 1932, sous la direction de Louis Martin-Chauffier (A. GIDE, Euvres complètes, Paris, Éditions de la Nouvelle Revue Française, vol. I, 1932). Les «magnifiques tomes» de ces Euvres seront évoqués une dernière fois dans la lettre $n^{\circ} 16$.

(5) Après le désert américain peuplé de «barbares», le désert égyptien figé en une «immobile terre», cette phrase ébauche le thème d'un troisième désert,générateur d'un isolement au moins aussi radical: le monde des lettres italien.

(6) «Roman» ou livre de «mémoires» dont nous savons peu de choses, mais dont la rumeur veut qu'il ait été commencé vers 1930 , puis vendu pour quelques lires, sous forme de manuscrit, à un bouquiniste italien (cf. A. AudOLI, «Omaggio a Agostino John Sinadino», cit., p. 45: «[risulta] disperso [...] l'autografo de Il fauno inginocchiato, un romanzo autobiografico che possiamo solo intuire: si vocifera che sia stato acquistato per pochi soldi da un bouquiniste milanese, e poi venduto a chi sa chi...»). En tous les cas, on retrouve ce titre dans la liste des ouvrages à paraitre des recueils de Sinadino depuis 1924, d'abord avec le sous-titre «III ${ }^{\circ}$ quaderno[del Dio dell'attimo]» (Il dio dell'attimo, 1924 et Poësies, 1929), puis avec le sous-titre: «IV ${ }^{\circ}$ Quaderno del Dio dell'attimo»(Vitae Subliminalis Aenigmata, 1934): le glissement dans la numéro- tation, à partir de 1934, est dû à la publication de Vitae Subliminalis Aenigmata, qui se substitue à lui comme troisième cahier du Dio dell'attimo. Ce livre «à paraître», mais toujours différé, est l'indice d'une véritable boulimie de projets, que n'arrêtent ni la vieillesse, ni l'isolement, ni les difficultés économiques.

(7) Ce recueil, dans lequel Sinadino investit ses dernières ressources, sera publié l'année suivante à Milan, par les éditions Corbaccio(Vitae Subliminalis Aenigmata, cit.).

(8) Rappelons que Gide a exprimé, au début des années 30 , sa sympathie à l'égard des communistes. Certaines «Pages de journal» publiées à partir du 1er juin 1932 dans la N.R.F. traduisent son espoir dans la nouvelle société de l'Union Soviétique, et laissent deviner son désir d'engagement; à la date du 13 mai 1931, on trouve par exemple dans le Journal la phrase suivante: «j' aimerais vivre assez pour voir le plan de la Russie réussir, et les États d'Europe contraints de s'incliner devant ce qu'ils s'obstinent à méconnaître» (A. GIDE, Journal 19261950, Paris, Gallimard, coll. «La Pléiade», 1997, p. 272). L'adhésion aux idées du communisme, qui s'accentue justement en 1933 - à la date de cette lettre - sera très éphémère: la déception de Gide est sensible dès 1936, lorsqu'il publie le Retour d'U.R.S.S.

(9) Significativement, Sinadino souhaite à son 
Comme vous voyez je me suis présenté à vous, bien humblement tel que vous m'avez connu, tel aussi que ces longues années de silence et d'angoisse m'ont fait, mais débordant toujours d'un même sentiment d'affection, qui me permet de souhaiter de votre part, cher et grand ami, un sentiment pareil, inestimable pour votre

Agostino J. Sinadino

16

\section{Gide à Sinadino ${ }^{1}$}

André Gide

I bis, rue Vaneau

Paris $7^{\mathrm{e}}$

Le 26 mars 1934

Monsieur SINADINO

c/o Edizioni Corbaccio

Via Principe Umberto 10

MILANO

Mon cher Sinadino,

Tout heureux de recevoir votre livre'; dont je n'ai lu encore et trop rapidement que quelques poèmes, ravi de les si bien comprendre et d'en pouvoir si bien apprécier le charme musical. Je n'avais jamais désespéré de recevoir de vous quelque message, ne parvenant pas à me persuader que vous puissiez avoir disparu pour toujours, mais quel plaisir me ferait une lettre de vous qui m'apprendrait un peu ce que vous êtes devenu.

Bien attentivement.

André Gide

correspondant une «toujours neuve inquiétude»: disposition d'esprit que Gide avait identifié, dans un essai sur Dostoïevski, comme le premier moteur de l'écriture; dans ce texte - à bien des égards un autoportrait - Gide insistait sur l'«insatisfaction de la chair», l'«inquiétude» et l'«anomalie» comme qualités premières de l'écrivain russe, et, partant, de tout écrivain (cf. A. GIDE, Dostoïevski, Paris, Plon, 1923). Sinadino avait justement dédié le premier cahier de Il dio dell'attimo (1910) «A I André Gide | "inquieto della diversità"».

16 Coll. part. Lettre dact., sign. aut., sans env. Papier libre. Un seul feuillet grand format, $210 \times 270 \mathrm{~mm}$.

(1) Lettre dont la traduction a été publiée par la revue $W U Z$, en même temps que les lettres $n^{\circ} 11$ et $\mathrm{n}^{\circ} 12$ (WUZ, cit., p. 43).

(2) Il s'agit du dernier recueil publié par A. J. Sinadino, conclusion de son parcours poétique en langue italienne: Vitae Subliminalis Aenigmata, cit. Ce sont les éditions milanaises Corbaccio, dirigées par Enrico dall'Oglio, qui se chargent de la publication de l'ouvrage, qui constitue une des rares incursions de cette maison d'édition dans le domaine de la poésie. Dall'Oglio possédait par ailleurs une librairie dans la Galleria Vittorio Emanuele, qui fut entre 1932 et 1936 un lieu de rencontre et d'échange pour tous les intellectuels de la capitale (cf. à ce propos A. GigLi MARCHETTI, Le edizioni Corbaccio. Storia di libri e di libertà, Milano, Franco Angeli, 2000). 
17

Sinadino à Gide

Milan, 16 avril [19]34

Via Leopardi 23

Tel: 19050

Vous seul - grand inoubliable - êtes à même d'expliquer l'étrange paralysie qui s'empare de l'être, empêchant la plume de traduire en messages réels les pensées, d'autant plus coutumières, que l'esprit aime adresser à l'Ami idéal et lointain...

Aussi, dois-je ajouter qu'un bref salut de Toscane, resté sans réponse, l'été passé, m'avait fait désespérer d'obtenir de vous des messages, sachant d'ailleurs vos énormes occupations et songeant à vos déplacements perpétuels.

Mais d'une chose je voudrais que vous soyez convaincu, mon cher Gide, de la primauté de cette amitié dont je suis le plus fier et toujours ému et dont une seule brève rencontre me fit connaître intensément le prix.

Vous êtes si bon de me demander ce que je fais. À part la crise dont la résultante est ce nouveau recueil de «poèmes» je travaille ou, plutôt, je suis hanté par un trop grand sujet de «confessions» suite à mon Faune agenouillé ${ }^{1}$ (pas paru), Le Prince terrestre $^{2}$ qui a comme point de départ le récit des trois tentations du Christ. Ce livre, vous seul pourriez l'écrire (en y ajoutant en sous-titre Scandale du Christ vrai) à la confusion de tous ces faux croyants dont les lettres sont infestées! Les magnifiques tomes de votre œuvre font mes délices ${ }^{3}$.

Gardez-moi votre précieuse amitié, cher grand Ami et songez, parfois, à votre très fidèle

Agostino J. Sinadino

17 B.L.J.D., 428-12. Lettre man. aut., sans env. Un feuillet grand format, $210 \times 265 \mathrm{~mm}$, recto verso. Corrections orthographiques: perpétuels pour: perpetuels; intensément pour: intensement. Correction de syntaxe: empêchant la plume de traduire pour: empêchant la plume à traduire. Autres remarques: une correction manuscrite:m'avait fait[e barré] désespérer. Signature soulignée.

(1) Ces mémoires à la troisième personne ont déjà été évoquées (lettre $\mathrm{n}^{\circ} 14$ ) sous leur titre italien, Il fauno inginocchiato.

(2) Nouveau projet, déjà annoncé dans la liste des ouvrages à paraître du recueil Vitae subliminalis aenigmata, sous un titre légèrement différent («Chronique du prince terrestre»). Parallèlement, Sinadino songeait à utiliser le titre en italien, pour le cinquième cahier du Dio dell'attimo: on retrouve dans cette même liste l'allusion à «Il principe terrestre. $V^{\circ}$ Quaderno del Dio dell'attimo». Le manuscrit ne fait pas partie des trois cahiers inédits récupérés par Ernesto Citro; en revanche, la représentation du Christ, la rédaction en langue française et l'hommage à Gide se retrouvent dans un texte bien postérieur (rédigé de 1947 à 1953), inclus dans ces cahiers inédits, et intitulé Cris de lumière. Evangile de N. S. l'Enfant chéri et divin selon Sinadinò: il n'est pas impossible que ce texte en soit la réécriture (cf. E. CiTRO, Agostino Jobn Sinadino e la poetica del simbolismo, Ravenna, Longo editore, coll. «Pleiadi», 1986, pp. 142-143).

(3) Par cette allusion aux Euvres complètes d'André Gide, en cours de publication à l'époque - à la date de la lettre, seuls les cinq premiers volumes, sur un ensemble de quinze, ont été imprimés - la correspondance se referme comme elle s'était ouverte, vingt-cing ans auparavant: l'admirateur de la Porte étroite est le premier lecteur des $\mathrm{Eu}$ vres publiées par la N.R.F. Et l'ami, l'auteur, le lecteur,finissent par se confondre dans l'adjectif que Sinadino choisit pour clore sa lettre: «très fidèle». 

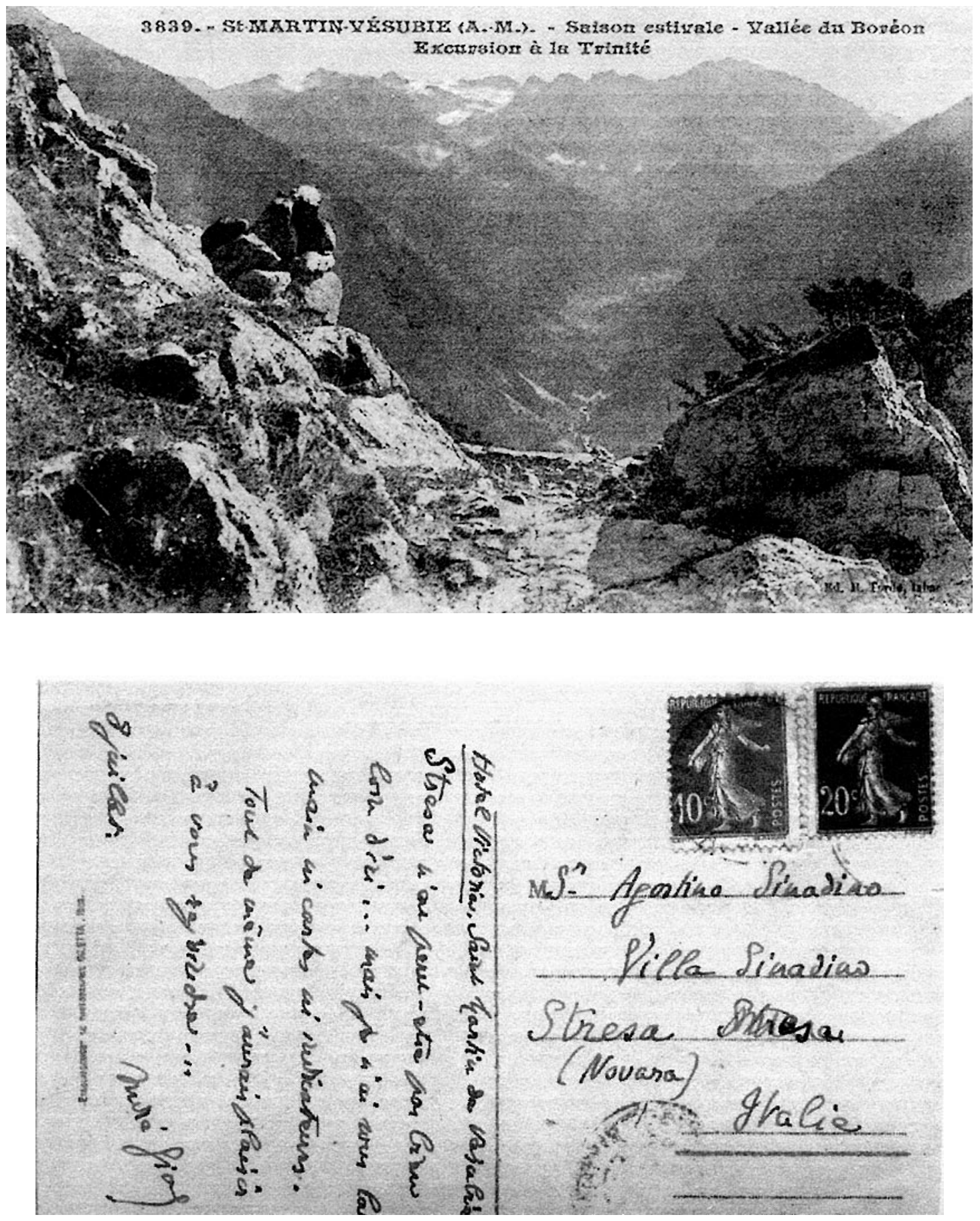

Carte postale d'André Gide à Agostino J. Sinadino (8 juillet 1923) reproduite avec l'aimable autorsation du critique et chasseur de manuscrits Armando Audoli. 